\title{
Estimating the unquenched strange quark mass from the lattice axial Ward identity
}

\author{
M. Göckeler, ${ }^{1}$ R. Horsley, ${ }^{2}$ A. C. Irving, ${ }^{3}$ D. Pleiter, ${ }^{4}$ P. E. L. Rakow, ${ }^{3}$ G. Schierholz,${ }^{4,5}$ H. Stüben, ${ }^{6}$ and J. M. Zanotti ${ }^{2}$ \\ (QCDSF-UKQCD Collaboration) \\ ${ }^{1}$ Institut für Theoretische Physik, Universität Regensburg, D-93040 Regensburg, Germany \\ ${ }^{2}$ School of Physics, University of Edinburgh, Edinburgh EH9 3JZ, United Kingdom \\ ${ }^{3}$ Department of Mathematical Sciences, University of Liverpool, Liverpool L69 3BX, United Kingdom \\ ${ }^{4}$ John von Neumann-Institut für Computing NIC, Deutsches Elektronen-Synchrotron DESY, D-15738 Zeuthen, Germany \\ ${ }^{5}$ Deutsches Elektronen-Synchrotron DESY, D-22603 Hamburg, Germany \\ ${ }^{6}$ Konrad-Zuse-Zentrum für Informationstechnik Berlin, D-14195 Berlin, Germany
}

(Received 13 January 2006; published 17 March 2006)

\begin{abstract}
We present a determination of the strange quark mass for two flavors $\left(n_{f}=2\right)$ of light dynamical quarks using the axial Ward identity. The calculations are performed on the lattice using $O(a)$ improved Wilson fermions and include a fully nonperturbative determination of the renormalization constant. In the continuum limit we find $m_{s}^{\overline{\mathrm{MS}}}(2 \mathrm{GeV})=111(6)(4)(6) \mathrm{MeV}$, using the force scale $r_{0}=0.467 \mathrm{fm}$, where the first error is statistical, the second and third are systematic due to the fit and scale uncertainties, respectively. Results are also presented for the light quark mass and the chiral condensate. The corresponding results are also given for $r_{0}=0.5 \mathrm{fm}$.
\end{abstract}

DOI: 10.1103/PhysRevD.73.054508

PACS numbers: $12.38 . \mathrm{Gc}$

\section{INTRODUCTION}

Lattice methods allow, in principle, an ab initio calculation of the fundamental parameters of QCD, among them the quark masses. Quarks are not asymptotic states of QCD and so quark masses need to be defined by a renormalization procedure,

$$
m_{q}^{\mathcal{S}}(M)=Z_{m}^{\mathcal{S}}(M) m_{q}^{\text {bare }},
$$

by giving the scheme $\mathcal{S}$ and scale $M$.

To convert the lattice results to continuum numbers, one needs control over the discretization errors and the matching relations between the lattice scheme and the continuum renormalization scheme $\mathcal{S}$. Discretisation errors can be kept small and manageable by employing an improved fermion action. But, still, the lattice numbers may show considerable cutoff dependence at present couplings, which requires that the calculations are done over a range of sufficiently small lattice spacings $a$, discretization errors then being removed by an extrapolation to $a=0$. The perturbative relations between renormalized quantities in the continuum and the bare lattice results are in almost all cases known to one-loop order only. Data show (for example [1]) that $O\left(\alpha_{s}^{2}\right)$ corrections can be large, $O(10 \%-$ $20 \%$ ) at spacings $a \approx 0.1 \mathrm{fm}$, which makes a nonperturbative calculation of the renormalization constants indispensible.

Many calculations of the strange quark mass, both with $n_{f}=2[2-4]$ and $n_{f}=2+1$ [5-8] flavors of sea quarks, employed perturbative renormalization of the bare quark mass and were restricted to lattice spacings $a \geqslant 0.1 \mathrm{fm}$. (Recent $n_{f}=2+1$ results [7] have finer lattice spacings.) These authors quote strange quark masses of $O(80) \mathrm{MeV}$, lying $15 \%-30 \%$ below the corresponding quenched results $[9,10]$.

In [11] we have presented an entirely nonperturbative (NP) calculation of the light quark masses based on the vector Ward identity (VWI), using nonperturbatively $O(a)$ improved Wilson fermions with $n_{f}=2$ flavors of dynamical quarks. The calculation was done at four different lattice spacings $0.065 \lessgtr a \lesssim 0.09 \mathrm{fm}$, which allowed us to perform a continuum extrapolation. We found a strange quark mass of $m_{s}^{\overline{\mathrm{MS}}}(2 \mathrm{GeV})=119(5)(8) \mathrm{MeV}$. A highlight and essential ingredient of the calculation was that we were able to compute the flavor singlet mass renormalization constant, which is needed in the VWI approach.

This result has been complemented recently by further studies in $[12,13]$ which also used NP determinations of the renormalization constant.

In this paper we present an independent calculation of the strange quark mass using the axial vector Ward identity (AWI), again for $n_{f}=2$ flavors of improved Wilson fermions. The AWI involves only nonsinglet quantities and thus provides an important test of our previous calculation.

The paper is organized as follows. As we shall be considering not only the $\overline{\mathrm{MS}}$ scheme, but also the RI'-MOM scheme (which is more convenient for a lattice calculation) we first discuss in Sec. II renormalization group invariants (RGIs), taking the quark mass as an example, and how to convert to them. We also collect together relevant formulas for the $\overline{\mathrm{MS}}$ scheme. Also discussed is the unit and scale we shall use - the $r_{0}$-force scale - and thus the relevant conversion factor to physical units. In Sec. III we compile some results from leading order (LO) and next to leading order (NLO) chiral perturbation theory $(\chi \mathrm{PT})$ and rewrite 
them in a form suitable for our calculation. Section IV describes some lattice details relevant for $O(a)$ improved fermions. This is followed in Sec. V by the nonperturbative computation of the renormalization constant. Relevant results for the $\mathrm{RI}^{\prime}-\mathrm{MOM}$ scheme are given, both for the lattice computation of $Z_{m}^{\mathrm{RI}-\mathrm{MOM}}$ and for the conversion to the RGI form. The section is concluded with a comparison of this result with the results obtained by other approaches (principally the tadpole-improved (TI) perturbation theory method). In Sec. VI results are given for the strange quark mass, first at finite lattice spacing, and then the continuum extrapolation is performed to give our final answer. Finally in the last section, Sec. VII, we compare our AWI result with the previously obtained VWI result and also with other recent mass determinations. In the appendix, tables of our raw data results for the quark mass are given.

\section{RENORMALIZATION GROUP INVARIANTS}

The "running" of the renormalized quark mass as the scale $M$ is changed is controlled by the $\beta$ and $\gamma$ functions in the renormalization group equation, defined by

$$
\begin{gathered}
\left.\beta^{\mathcal{S}}\left(g^{\mathcal{S}}(M)\right) \equiv \frac{\partial g^{\mathcal{S}}(M)}{\partial \log M}\right|_{\text {bare }}, \\
\left.\gamma_{m}^{\mathcal{S}}\left(g^{\mathcal{S}}(M)\right) \equiv \frac{\partial \log Z_{m}^{\mathcal{S}}(M)}{\partial \log M}\right|_{\text {bare }},
\end{gathered}
$$

where the bare parameters are held constant. These functions are given perturbatively as power series expansions in the coupling constant,

$$
\begin{aligned}
& \beta^{\mathcal{S}}(g)=-b_{0} g^{3}-b_{1} g^{5}-b_{2}^{\mathcal{S}} g^{7}-b_{3}^{\mathcal{S}} g^{9}-\ldots, \\
& \gamma_{m}^{\mathcal{S}}(g)=d_{m 0} g^{2}+d_{m 1}^{\mathcal{S}} g^{4}+d_{m 2}^{\mathcal{S}} g^{6}+d_{m 3}^{\mathcal{S}} g^{8}+\ldots
\end{aligned}
$$

The first two coefficients of the $\beta$-function and first coefficient of the $\gamma_{m}$ function are scheme independent,

$$
b_{0}=\frac{1}{(4 \pi)^{2}}\left(11-\frac{2}{3} n_{f}\right), \quad b_{1}=\frac{1}{(4 \pi)^{4}}\left(102-\frac{38}{3} n_{f}\right) .
$$

and

$$
d_{m 0}=-\frac{8}{(4 \pi)^{2}},
$$

while all others depend on the scheme chosen.

We may immediately integrate Eq. (2) to obtain

$$
\begin{aligned}
\frac{M}{\Lambda^{\mathcal{S}}}= & {\left[b_{0} g^{\mathcal{S}}(M)^{2}\right]^{b_{1} / 2 b_{0}^{2}} \exp \left[\frac{1}{2 b_{0} g^{\mathcal{S}}(M)^{2}}\right] } \\
& \times \exp \left\{\int_{0}^{g^{\mathcal{S}}(M)} d \xi\left[\frac{1}{\beta^{\mathcal{S}}(\xi)}+\frac{1}{b_{0} \xi^{3}}-\frac{b_{1}}{b_{0}^{2} \xi}\right]\right\} .
\end{aligned}
$$

The renormalization group invariant quark mass ${ }^{1}$ is defined

\footnotetext{
${ }^{1}$ Analogous definitions hold for other quantities which depend on the scheme and scale chosen.
}

from the renormalized quark mass as

$$
\begin{aligned}
m_{q}^{\mathrm{RGI}} & \equiv \Delta Z_{m}^{\mathcal{S}}(M) m^{\mathcal{S}}(M)=\Delta Z_{m}^{\mathcal{S}}(M) Z_{m}^{\mathcal{S}}(M) m_{q}^{\text {bare }} \\
& \equiv Z_{m}^{\mathrm{RGI}} m_{q}^{\text {bare }},
\end{aligned}
$$

where

$$
\begin{aligned}
{\left[\Delta Z_{m}^{\mathcal{S}}(M)\right]^{-1}=} & {\left[2 b_{0} g^{\mathcal{S}}(M)^{2}\right]^{-\left(d_{m 0} / 2 b_{0}\right)} } \\
& \times \exp \left\{\int_{0}^{g^{\mathcal{S}}(M)} d \xi\left[\frac{\gamma_{m}^{\mathcal{S}}(\xi)}{\beta^{\mathcal{S}}(\xi)}+\frac{d_{m 0}}{b_{0} \xi}\right]\right\}
\end{aligned}
$$

and so the integration constant upon integrating Eq. (2) is given by $\Lambda^{\mathcal{S}}$, and similarly from Eq. (3) the integration constant is $m_{q}^{\mathrm{RGI}} . \Lambda^{\mathcal{S}}$ and $m_{q}^{\mathrm{RGI}}$ are thus independent of the scale. (Note that although the functional form of $\Delta Z_{m}^{\mathcal{S}}(M)$ is fixed, the absolute value is not; conventions vary for its definition.) Also for a scheme change $\mathcal{S} \rightarrow \mathcal{S}^{\prime}$ (it is now sufficient to take them at the same scale) given by

$$
g^{\mathcal{S}^{\prime}}=G\left(g^{\mathcal{S}}\right)=g^{\mathcal{S}}\left(1+\frac{1}{2} t_{1}\left(g^{\mathcal{S}}\right)^{2}+\ldots\right)
$$

then $m_{q}^{\mathrm{RGI}}$ remains invariant, while $\Lambda$ changes as $\Lambda^{\mathcal{S}^{\prime}}=$ $\Lambda^{\mathcal{S}} \exp \left(t_{1} /\left(2 b_{0}\right)\right)$. Note also that analytic expressions for the integrals in Eq. (8) or Eq. (9) can be found for low orders, for example, to two loops we have

$$
\begin{aligned}
\Delta Z_{m}^{\mathcal{S}}(M)= & {\left[2 b_{0}\left(g^{\mathcal{S}}(M)\right)^{2}\right]^{d_{m 0} / 2 b_{0}} } \\
& \times\left[1+\frac{b_{1}}{b_{0}}\left(g^{\mathcal{S}}(M)\right)^{2}\right]^{\left(b_{0} d_{m 1}^{\mathcal{S}}-b_{1} d_{m 0}\right) / 2 b_{0} b_{1}} .
\end{aligned}
$$

Thus we have a convenient splitting of the problem into two parts: a number, $m_{q}^{\mathrm{RGI}}$, which involves a nonperturbative computation, and is the goal of this paper and, if desired, an evaluation of $\Delta Z_{m}^{\mathcal{S}}$ which allows the running quark mass to be given in a renormalization scheme $\mathcal{S}$.

In the remainder of this section we discuss the evaluation of $\Delta Z_{m}^{\mathcal{S}}$ in the $\overline{\mathrm{MS}}$-scheme, which is conventionally used, and for which four coefficients in the perturbative expansion are known. For the $\beta$ function we have [14-16],

$$
\begin{aligned}
b_{2}^{\overline{\mathrm{MS}}}= & \frac{1}{(4 \pi)^{6}}\left[\frac{2857}{2}-\frac{5033}{18} n_{f}+\frac{325}{54} n_{f}^{2}\right], \\
b_{3}^{\overline{\mathrm{MS}}}= & \frac{1}{(4 \pi)^{8}}\left[\frac{149753}{6}+3564 \zeta_{3}-\left(\frac{1078361}{162}\right.\right. \\
& \left.+\frac{6508}{27} \zeta_{3}\right) n_{f}+\left(\frac{50065}{162}+\frac{6472}{81} \zeta_{3}\right) n_{f}^{2} \\
& \left.+\frac{1093}{729} n_{f}^{3}\right],
\end{aligned}
$$

and for the $\gamma_{m}$ function [17,18], 


$$
\begin{aligned}
d_{m 1}^{\overline{\mathrm{MS}}}= & -\frac{1}{(4 \pi)^{4}}\left[\frac{404}{3}-\frac{40}{9} n_{f}\right], \\
d_{m 2}^{\overline{\mathrm{MS}}}= & -\frac{1}{(4 \pi)^{6}}\left[2498-\left(\frac{4432}{27}+\frac{320}{3} \zeta_{3}\right) n_{f}-\frac{280}{81} n_{f}^{2}\right], \\
d_{m 3}^{\overline{\mathrm{MS}}}= & -\frac{1}{(4 \pi)^{8}}\left[\frac{4603055}{81}+\frac{271360}{27} \zeta_{3}-17600 \zeta_{5}\right. \\
& -\left(\frac{183446}{27}+\frac{68384}{9} \zeta_{3}-1760 \zeta_{4}-\frac{36800}{9} \zeta_{5}\right) n_{f} \\
& +\left(\frac{10484}{243}+\frac{1600}{9} \zeta_{3}-\frac{320}{3} \zeta_{4}\right) n_{f}^{2} \\
& \left.\left.-\left(\frac{664}{243}-\frac{128}{27} \zeta_{3}\right) n_{f}^{3}\right)\right],
\end{aligned}
$$

with $\zeta_{3}=1.20206 \ldots, \quad \zeta_{4}=1.08232 \ldots$ and $\zeta_{5}=$ $1.03693 \ldots, \zeta$ being the Riemann zeta function.

This scheme is a manifestly perturbative scheme and so should be used at a high enough scale $M \equiv \mu$ so that perturbation theory is reliable. Computing $\left[\Delta Z_{m}^{\overline{\mathrm{MS}}}(\mu)\right]^{-1}$ involves first solving Eq. (7) for $g^{\overline{\mathrm{MS}}}$ (as a function of $\mu / \Lambda^{\overline{\mathrm{MS}}}$ ) and then evaluating Eq. (9). Practically we expand the $\beta$ and $\gamma$ functions to the appropriate order and then numerically evaluate the integrals. The final results are given in Fig. 1.

Conventionally light quark masses are defined at a scale of $\mu=2 \mathrm{GeV}$, which means giving a value for $\Lambda^{\overline{\mathrm{MS}}}$ in $\mathrm{MeV}$. We set the scale here by using the "force scale" $r_{0}$, which means first changing from the $\Lambda^{\overline{\mathrm{MS}}}$ unit to the $r_{0}$ unit. From [19] (see also [20]), we use the value

$$
r_{0} \Lambda^{\overline{\mathrm{MS}}}=0.617(40)(21) .
$$

The $r_{0}$ scale in $\mathrm{MeV}$ still needs to be set. A popular choice

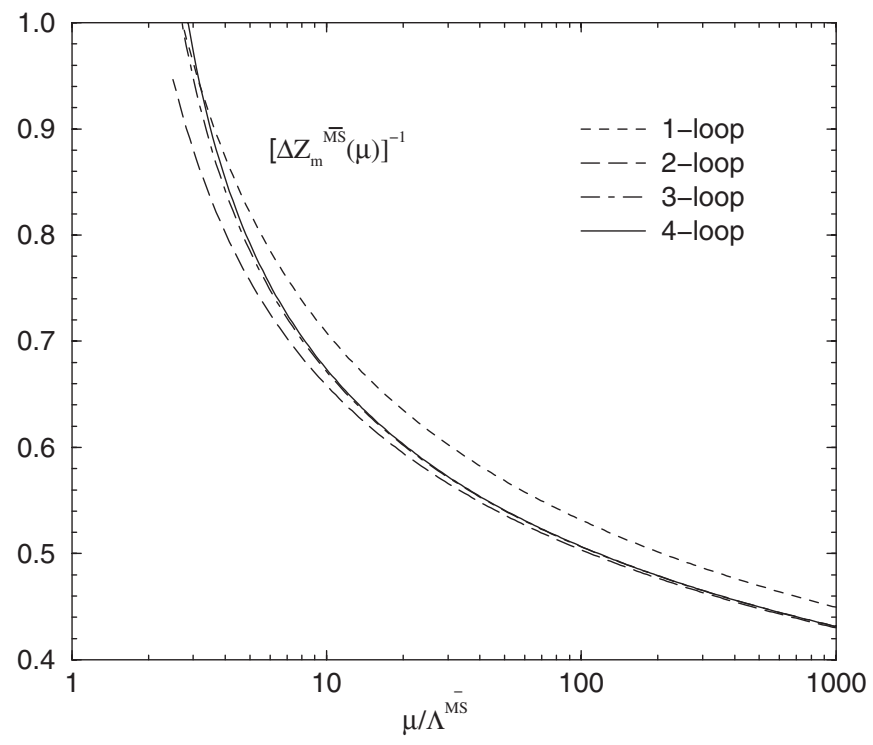

FIG. 1. One-, two-, three- and four-loop results for $\left[\Delta Z_{m}^{\overline{\mathrm{MS}}}(\mu)\right]^{-1}$ in units of $\Lambda^{\overline{\mathrm{MS}}}$.
TABLE I. Values of $\left[\Delta Z_{m}^{\overline{\mathrm{MS}}}(\mu)\right]^{-1}$ at $\mu=2 \mathrm{GeV}$. The errors are a reflection of the errors in Eq. (14).

\begin{tabular}{lcccc}
\hline \hline$r_{0}$ & one-loop & two-loop & three-loop & four-loop \\
\hline $0.5 \mathrm{fm}$ & $0.735(11)$ & $0.682(10)$ & $0.698(11)$ & $0.700(11)$ \\
$0.467 \mathrm{fm}$ & $0.745(12)$ & $0.690(10)$ & $0.707(12)$ & $0.711(12)$ \\
\hline \hline
\end{tabular}

is

$$
r_{0}=0.5 \mathrm{fm} \equiv 1 /(394.6 \mathrm{MeV}),
$$

which is useful when making comparisons with other results for the quark mass. Alternatively from a fit to the dimensionless nucleon mass $r_{0} m_{N}$ using results obtained by the CP-PACS, JLQCD and QCDSF-UKQCD collaborations, following [21] we found a scale of

$$
r_{0}=0.467(33) \mathrm{fm} \equiv 1 /(422.5(29.9) \mathrm{MeV}) .
$$

Similar results were obtained in $[22,23]$. The $0.033 \mathrm{fm}$ error in the $r_{0}=0.467 \mathrm{fm}$ estimate is roughly equal to the difference between the two $r_{0}$ values. As these different $r_{0}$ values give an idea of the uncertainties involved in setting the scale we shall derive results using both values of $r_{0}$ and regard this as giving an estimate of a possible scale systematic error. The different values of $r_{0}$ in Eqs. (15) and (16) give $\Lambda^{\overline{\mathrm{MS}}}=243(16)(8) \mathrm{MeV}$ and 261(17)(9) MeV, respectively.

Results for $\left[\Delta Z_{m}^{\overline{\mathrm{MS}}}\right]^{-1}$ at $\mu=2 \mathrm{GeV}$ are given in Table I. At $\mu=2 \mathrm{GeV}$ we have $\mu / \Lambda^{\overline{\mathrm{MS}}} \approx 8$, and it seems that already at this value we have a rapidly converging series in loop orders. Indeed, only going from one loop to two loops gives a significant change in $\left[\Delta Z_{m}^{\overline{\mathrm{MS}}}\right]^{-1}$ of order $8 \%$. From two loops to three loops we have about $2 \%$. The difference between the three-loop and four-loop results is $O\left(\frac{1}{2} \%\right)$. So if we are given $m_{q}^{\mathrm{RGI}}$, and we wish to find the quark mass in the $\overline{\mathrm{MS}}$ scheme at a certain scale, we shall use the four-loop result from Eq. (9) as shown in Fig. 1.

\section{CHIRAL PERTURBATION THEORY}

Chiral perturbation theory $(\chi \mathrm{PT})$ gives the LO and NLO result $^{2}[25,26]$ for $n_{f}$ degenerate sea quarks of

$$
\begin{aligned}
\left(\frac{m_{p s}^{A B}}{4 \pi f_{0}}\right)^{2}= & \chi_{A B}\left[1+\chi_{S} n_{f}\left(2 \alpha_{6}-\alpha_{4}\right)+\chi_{A B}\left(2 \alpha_{8}-\alpha_{5}\right)\right. \\
& \left.+\frac{1}{n_{f}} \frac{\chi_{A}\left(\chi_{S}-\chi_{A}\right) \ln \chi_{A}-\chi_{B}\left(\chi_{S}-\chi_{B}\right) \ln \chi_{B}}{\chi_{B}-\chi_{A}}\right],
\end{aligned}
$$

where the first term on the right-hand side (RHS) is the LO term, the NLO terms being the remaining terms and $\alpha_{i}$ are the low energy chiral constants (LECs) evaluated at the scale $\Lambda_{\chi}=4 \pi f_{\pi}$. With our conventions for the pion decay

\footnotetext{
${ }^{2}$ The NNLO result has recently been constructed in [24].
} 
constant in the chiral limit, $f_{0}$, we have $f_{\pi}=92.4 \mathrm{MeV}$ so that $\Lambda_{\chi} \approx 1160 \mathrm{MeV}$.

$\chi_{A B}$ are related to the quark masses by [26],

$$
\chi_{A B}=\frac{B_{0}^{\mathcal{S}}\left(m_{A}+m_{B}\right)^{\mathcal{S}}}{\left(4 \pi f_{0}\right)^{2}}, \quad A, B \in\left\{V_{1}, V_{2}, S\right\}
$$

where $m_{S}$ is the sea quark mass and $m_{V_{i}}, i=1,2$ are the (possibly) nondegenerate valence quark masses. In particular we have

$$
\chi_{A B}=\frac{1}{2}\left(\chi_{A}+\chi_{B}\right), \quad \text { where } \chi_{A} \equiv \chi_{A A} .
$$

Furthermore, in Eq. (18) $B_{0}^{\mathcal{S}}$ is related to the chiral condensate by

$$
B_{0}^{\mathcal{S}}=-\frac{1}{f_{0}^{2}}\langle\bar{q} q\rangle^{\mathcal{S}}
$$

Apart from $f_{\pi}$, none of the other LECs [here $B_{0}$, or $\langle\bar{q} q\rangle$, and $\alpha_{4}, \alpha_{5}, \alpha_{6}, \alpha_{8}$ in Eq. (17)] are well determined. Typical values are (for $n_{f}=3$ ), $\alpha_{4}=-0.76(60), \alpha_{5}=$ 0.5(6), $\alpha_{6}=-0.5(4), \alpha_{8}=0.76(40)$ (as compiled in [27]) giving

$$
\begin{gathered}
2 \alpha_{6}-\alpha_{4} \approx-0.24, \quad 2 \alpha_{8}-\alpha_{5} \approx 1.02, \\
\langle\bar{q} q\rangle^{\overline{\mathrm{MS}}}(2 \mathrm{GeV}) \approx-(267 \mathrm{MeV})^{3},
\end{gathered}
$$

(the $\langle\bar{q} q\rangle$ result is taken from [28]).

$m_{p s}^{A B}$ in Eq. (17) is the pseudoscalar mass (with $A, B \in$ $\left.\left\{V_{1}, V_{2}\right\}\right)$ implicitly depending on the sea quark mass $m_{S}$. Again for degenerate valence quarks, we write $m_{p s}^{A} \equiv m_{p s}^{A A}$. Note that we also numerically allow for a valence quark mass to be equal to the sea quark mass, so, for example, we can write $m_{p s}^{S}$.

We shall assume Eq. (17) as the basic functional form for the relation between the quark mass and the pseudoscalar mass in the following. As expected this equation is symmetric under an interchange of the two valence quarks. However it does not have the most general structure allowed by this symmetry.

Equation (17) is not very convenient for comparing with numerical results for a variety of reasons [see Sec. VI A and Eqs. (57) and (58)]. First, the quantities we measure are not $\chi_{A B}, m_{p s}^{A B}$ but only proportional to them,

$$
\chi_{A B}=c_{\chi} y_{A B}, \quad \frac{m_{p s}^{A B}}{4 \pi f_{0}}=c_{m} M_{p s}^{A B},
$$

where $y_{A B}$ and $M_{p s}^{A B}$ are new variables. Substituting these into Eq. (17) simply shifts the coefficients of the various terms (including the 1 and $1 / n_{f}$ terms), while the structural form of this equation remains the same. Note that this includes the cases where $c_{\chi}$ and $c_{m}$ are functions of the quark mass, e.g.

$$
\begin{aligned}
& c_{\chi} \rightarrow c_{\chi}^{(0)}+c_{\chi}^{S(1)} y_{S}+c_{\chi}^{A B(1)} y_{A B}+\ldots, \\
& c_{m} \rightarrow c_{m}^{(0)}+c_{m}^{S(1)} y_{S}+c_{m}^{A B(1)} y_{A B}+\ldots
\end{aligned}
$$

Second, we prefer to work with a function of the pseudoscalar mass rather than the quark mass, so we invert Eq. (17). This gives a functional form, which we choose to write as

$$
\begin{aligned}
\frac{y_{A B}}{\left(M_{p s}^{A B}\right)^{2}}= & c_{a}+\left(\frac{c_{b}-c_{d}\left(1+\ln c_{a}\right)}{c_{a}}\right) y_{S} \\
& +\left(\frac{c_{c}+c_{d}\left(1+2 \ln c_{a}\right)}{c_{a}}\right) y_{A B} \\
& -\left(\frac{c_{d}}{c_{a}}\right) \frac{y_{A}\left(y_{S}-y_{A}\right) \ln y_{A}-y_{B}\left(y_{S}-y_{B}\right) \ln y_{B}}{y_{B}-y_{A}} .
\end{aligned}
$$

Setting $A=B=V$ this equation reduces to the degenerate valence case (and finally setting $V \equiv S$ gives the sea quark case). These sets of equations may be (once) iterated to produce $y_{A B} /\left(M_{p s}^{A B}\right)^{2}$ as a function of $\left(M_{p s}^{A B}\right)^{2}$ and $\left(M_{p s}^{S}\right)^{2}$. Relevant later will be the case of degenerate valence quarks when we have

$$
\begin{aligned}
\frac{y_{V}}{\left(M_{p s}^{V}\right)^{2}}= & c_{a}+c_{b}\left(M_{p s}^{S}\right)^{2}+c_{c}\left(M_{p s}^{V}\right)^{2} \\
& +c_{d}\left(\left(M_{p s}^{S}\right)^{2}-2\left(M_{p s}^{V}\right)^{2}\right) \ln \left(M_{p s}^{V}\right)^{2},
\end{aligned}
$$

which explains our original choice of the $c_{a}, c_{i}(i=b, c, d)$ coefficients in Eq. (24). So, as mentioned previously, we see that determining these coefficients from Eq. (25), which only needs degenerate valence quark masses, is sufficient to find the results for nondegenerate quark masses, Eq. (24).

Numerically we shall find that higher order terms in $\chi \mathrm{PT}$ are small, i.e. $c_{a} \gg\left|c_{i}\right| M_{p s}^{2}(i=b, c, d)$ and thus all these manipulations are justified.

The relation between $c_{\chi}, c_{m}$ and $c_{a}$ is, from the $\mathrm{LO}$ term

$$
\frac{c_{m}^{2}}{c_{\chi}}=c_{a} .
$$

At NLO we have in addition

$$
c_{m}^{2}=n_{f} \frac{c_{d}}{c_{a}},
$$

and for the remaining LECs the relationships

$$
\begin{aligned}
& 2 \alpha_{6}-\alpha_{4}=\frac{1}{n_{f}^{2}}\left[1+\ln n_{f}-\frac{c_{b}}{c_{d}}+\ln \frac{c_{d}}{c_{a}}\right], \\
& 2 \alpha_{8}-\alpha_{5}=-\frac{1}{n_{f}}\left[1+2 \ln n_{f}+\frac{c_{c}}{c_{d}}+2 \ln \frac{c_{d}}{c_{a}}\right] .
\end{aligned}
$$


We write the results only for the case when $c_{m}^{(1)}=c_{\chi}^{(1)}=0$ [see Eq. (23)]. This is sufficient for the later estimation of the LECs in Sec. VI, as we shall be using continuum results. ${ }^{3}$

Finally we have to find a formula for the strange quark mass. We first note that we have

(i) Two degenerate sea quarks $m_{S} \equiv m_{u d}=\frac{1}{2} \times$ $\left(m_{u}+m_{d}\right)$

(ii) Three possible valence quarks, $m_{u}, m_{d}$ and $m_{s}$ (where " $s$ " denotes the strange quark). We write $m_{u}=m_{u d}-\Delta m_{u d}$ and $m_{d}=m_{u d}+\Delta m_{u d}$ where $\Delta m_{u d}=\left(m_{d}-m_{u}\right) / 2$ is proportional to the difference between the down and up quark masses.

Inputting meson data we use the $K^{+}(u \bar{s})$, where we set $A=$ $u, B=s$ in Eq. (24), $K^{0}(d \bar{s})$, where we have $A=d, B=s$, and together with the $\pi^{+}(u \bar{d})$, with $A=u, B=d$, gives after some algebra the result

$$
\begin{aligned}
y_{s}= & c_{a}\left[M_{K^{+}}^{2}+M_{K^{0}}^{2}-M_{\pi^{+}}^{2}\right]+\left(c_{b}-c_{d}\right)\left[M_{K^{+}}^{2}+M_{K^{0}}^{2}\right] M_{\pi^{+}}^{2} \\
& +\frac{1}{2}\left(c_{c}+c_{d}\right)\left[M_{K^{+}}^{2}+M_{K^{0}}^{2}\right]^{2}-\left(c_{b}+c_{c}\right) M_{\pi^{+}}^{4} \\
& -c_{d}\left[M_{K^{+}}^{2}+M_{K^{0}}^{2}\right]\left[M_{K^{+}}^{2}+M_{K^{0}}^{2}-M_{\pi^{+}}^{2}\right] \\
& \times \ln \left(M_{K^{+}}^{2}+M_{K^{0}}^{2}-M_{\pi^{+}}^{2}\right)+c_{d} M_{\pi^{+}}^{4} \ln M_{\pi^{+}}^{2}+\cdots,
\end{aligned}
$$

for the strange quark and

$$
y_{u d}=c_{a} M_{\pi^{+}}^{2}+\left(c_{b}+c_{c}\right) M_{\pi^{+}}^{4}-c_{d} M_{\pi^{+}}^{4} \ln M_{\pi^{+}}^{2}+\cdots,
$$

for the light quark, where the $\cdots$ include higher order terms in $\chi \mathrm{PT}$ (i.e. NNLO) and terms of $O\left(\left(\Delta m_{u d}\right)^{2}\right)$.

The results of Eqs. (29) and (30) are valid for "pure" QCD. To include electromagnetic effects, we use Dashen's theorem which says that if electromagnetic effects are the only source of breaking of isospin symmetry (i.e. $m_{u}=$ $\left.m_{d}\right)$, the leading electromagnetic contribution to $m_{K^{+}}^{2}$ and $m_{\pi^{+}}^{2}$ are equal while $m_{\pi^{0}}^{2}$ and $m_{K^{0}}^{2}$ are unaffected (see e.g. [29]). Thus the masses in Eqs. (29) and (30) may be written as [30]

$$
\begin{aligned}
& m_{K^{+}}^{2}=\left(m_{K^{+}}^{\mathrm{EXPT}}\right)^{2}-\left(m_{\pi^{+}}^{\mathrm{EXPT}}\right)^{2}+\left(m_{\pi^{0}}^{\mathrm{EXPT}}\right)^{2}, \\
& m_{K^{0}}^{2}=\left(m_{K^{0}}^{\mathrm{EXPT}}\right)^{2}, \quad m_{\pi^{+}}^{2}=m_{\pi^{0}}^{2}=\left(m_{\pi^{0}}^{\mathrm{EXPT}}\right)^{2},
\end{aligned}
$$

where $m_{K^{+}}, m_{K^{0}}, m_{\pi^{+}}$and $m_{\pi^{0}}$ are the "pure" $\mathrm{QCD}$ numbers, while $m_{K^{+}}^{\text {EXT }}, m_{K^{0}}^{\text {EXPT }}, m_{\pi^{+}}^{\text {EXPT }}$ and $m_{\pi^{0}}^{\text {EXPT }}$ are the experimentally observed numbers. Dashen's theorem has corrections $O\left(\alpha_{\mathrm{QED}} m_{q}\right)$ from higher order terms in $\chi \mathrm{PT}$, estimates vary as to the magnitude of this correction

\footnotetext{
${ }^{3}$ For the more general case in Eqs. (26) $-(28) c_{m}, c_{\chi}$ are replaced by $c_{m}^{(0)}$ and $c_{\chi}^{(0)}$, respectively, together with additional terms on the RHS of Eq. (28) of $-1 /\left(n_{f}\left(c_{\chi}^{(0)}\right)^{2}\right) \times$ $\left(c_{\chi}^{S(1)}-2 c_{m}^{S(1)} / c_{m}^{(0)}\right)$ and $-1 /\left(c_{\chi}^{(0)}\right)^{2}\left(c_{\chi}^{A B(1)}-2 c_{m}^{A B(1)} / c_{m}^{(0)}\right)$ for the second and third equations, respectively.
}

[31,32], see [30,33] for a discussion. See also [34-36]. Recent results from the lattice approach $[37,38]$ seem to indicate only a mild breaking of Dashen's theorem.

Note that to LO in $\chi \mathrm{PT}$ using experimental values of the $\pi$ and $K$ masses, namely,

$$
\begin{array}{ll}
m_{\pi^{+}}^{\text {EXPT }}=139.6 \mathrm{MeV} & m_{\pi^{0}}^{\text {EXPT }}=135.0 \mathrm{MeV}, \\
m_{K^{+}}^{\text {EXPT }}=493.7 \mathrm{MeV} & m_{K^{0}}^{\text {EXPT }}=497.7 \mathrm{MeV},
\end{array}
$$

we have the result

$$
\begin{aligned}
\frac{m_{s}^{\mathcal{S}}(M)}{m_{u d}^{\mathcal{S}}(M)} & =\frac{m_{K^{+}}^{2}+m_{K^{0}}^{2}-m_{\pi^{+}}^{2}}{m_{\pi^{+}}^{2}} \\
& =\frac{\left(m_{K^{+}}^{\mathrm{EXPT}}\right)^{2}+\left(m_{K^{0}}^{\mathrm{EXPT}}\right)^{2}-\left(m_{\pi^{+}}^{\mathrm{EXPT}}\right)^{2}}{\left(m_{\pi^{0}}^{\mathrm{EXT}}\right)^{2}} \approx 25.9,
\end{aligned}
$$

independent of the value of $c_{a}$. So if we are in or close to this regime, once we have determined the strange quark mass, this immediately gives an estimate of the light quark mass. Incorporating the NLO terms needs a determination of all the $c_{a}$ and $c_{i}(i=b, c, d)$ coefficients in Eqs. (29) and (30), and gives the results in Sec. VIB.

\section{THE LATTICE APPROACH}

Here we shall derive results for the unquenched $\left(n_{f}=2\right)$ strange quark mass using the axial Ward identity. All our numerical computations are done with degenerate valence quark masses.

The starting point is the AWI; in the continuum we have the renormalized relation

$$
\partial_{\mu} \mathcal{A}_{\mu}^{\mathcal{R}}=2 m_{q}^{\mathcal{S}}(M) \mathcal{P}^{\mathcal{S}}(M),
$$

where $\mathcal{A}^{\mathcal{R}}$ and $\mathcal{P}^{\mathcal{S}}$ are the renormalized (in scheme $\mathcal{S}$ ) axial current and pseudoscalar density, respectively. In the (bare) lattice theory the current quark masses are also defined via the equivalent $\mathrm{AWI}^{4}$

$$
\partial_{\mu}^{\mathrm{LAT}} \mathcal{A}_{\mu}=2 \tilde{m}_{q} \mathcal{P}+O\left(a^{2}\right),
$$

where $\mathcal{A}$ and $\mathcal{P}$ are the $O(a)$ improved unrenormalized axial current and pseudoscalar density

$$
\begin{aligned}
\mathcal{A}_{\mu} & =\left(1+b_{A} a m_{q}\right)\left(A_{\mu}+c_{A} a \partial_{\mu}^{\mathrm{LAT}} P\right), \\
\mathcal{P} & =\left(1+b_{P} a m_{q}\right) P,
\end{aligned}
$$

with

$$
A_{\mu}=\bar{q} \gamma_{\mu} \gamma_{5} q, \quad P=\bar{q} \gamma_{5} q .
$$

Wilson-type fermions allow several different definitions of the quark mass. We denote the (bare) quark mass defined from the AWI with a tilde, $a \tilde{m}_{q}$, while that from the VWI is given by

\footnotetext{
${ }^{4} \partial_{\mu}^{\mathrm{LAT}}$ is the symmetric lattice derivative, conventionally chosen to be $\left(\partial_{\mu}^{\mathrm{LAT}} f\right)(x)=[f(x+a \hat{\mu})-f(x-a \hat{\mu})] /(2 a)$, where $\hat{\mu}$ is a unit vector in the $\mu$ direction.
} 


$$
a m_{q}=\frac{1}{2}\left(\frac{1}{\kappa_{q}}-\frac{1}{\kappa_{q c}^{S}}\right),
$$

where $\kappa_{q}$ is the Wilson hopping parameter, defining the quark mass (both sea and valence). The critical sea quark hopping parameter, $\kappa_{q c}^{S}$, is defined for fixed $\beta \equiv 6 / g_{0}^{2}$ (where $g_{0}$ is the lattice coupling) by the vanishing of the pseudoscalar mass, ${ }^{5}$ i.e. $\left.m_{p s}\right|_{\kappa_{q}=\kappa_{q c}^{S}}=0$.

Returning to Eq. (36), $c_{A}$ is known nonperturbatively [39], but not $b_{A}$ and $b_{P}$. However results using one-loop perturbation theory [40], or for nonperturbative quenched QCD [41], show that the difference $b_{A}-b_{P}$ is small (there is however an increase between the perturbative and quenched nonperturbative results). Multiplying by $a m_{q}$ thus gives a correction of perhaps half a percent, which with our present level of accuracy we can ignore.

Forming lattice correlation functions means that the quark mass can be defined and determined from the ratio ${ }^{6}$

$$
\begin{aligned}
a \tilde{m}_{q} \stackrel{t \gg 0}{=} \frac{\left\langle\partial_{4}^{\mathrm{LAT}} \mathcal{A}_{4}(t) \mathcal{O}(0)\right\rangle}{2\langle\mathcal{P}(t) \mathcal{O}(0)\rangle} \approx & \frac{\left\langle\partial_{4}^{\mathrm{LAT}} A_{4}(t) \mathcal{O}(0)\right\rangle}{2\langle\mathcal{P}(t) \mathcal{O}(0)\rangle}+c_{A} a \\
& \times \frac{\left\langle\partial_{4}^{2 \mathrm{LAT}} P(t) \mathcal{O}(0)\right\rangle}{2\langle\mathcal{P}(t) \mathcal{O}(0)\rangle}+O\left(a^{2}\right) \\
\equiv & a \tilde{m}_{q}^{(0)}+c_{A} a \tilde{m}_{q}^{(1)}+O\left(a^{2}\right),
\end{aligned}
$$

where $\approx$ in the second equation signifies that we have dropped the correction factor $1+\left(b_{A}-b_{P}\right) a m_{q} . \mathcal{O}$ is an operator with a nonzero overlap with the pseudoscalar particle. We choose it here to be $P^{\text {smeared }}$, where we have used Jacobi smearing (see the appendix) on the operator.

The parameter space spanned in our numerical simulations is given in Table II. The notation is standard for the parameters of the action, see for example [43]. (The critical Wilson hopping parameters, $\kappa_{q c}^{S}$, have been determined in [11] for each $\beta$.)

In the appendix we list our $\kappa_{q}$ for each $\kappa_{q}^{S}$ together with the corresponding partially quenched $a m_{p s}$ and bare AWI quark masses $a \tilde{m}_{q}^{(0)}, a \tilde{m}_{q}^{(1)}$ and $a \tilde{m}_{q}$.

\section{RENORMALIZATION}

\section{A. Generalities}

Imposing the AWI on the lattice, Eq. (35), up to cutoff effects means that the axial current as well as the pseudoscalar density and the quark mass must be renormalized. Thus we have

\footnotetext{
${ }^{5}$ We shall suppress the " $V$ " superscript on the pseudoscalar mass and only include an " $S$ " superscript where necessary.

${ }^{6}$ Note that to reduce noise, derivatives of operators on the lattice are taken as compact as possible, consistent with the given symmetry. Thus we use, no $\mu$ summation, $\left(\partial_{\mu}^{2 \mathrm{LAT}} f\right)(x)=[f(x+$ $a \hat{\mu})-2 f(x)+f(x-a \hat{\mu})] /(2 a)^{2}=\left(\partial_{\mu}^{\mathrm{LAT}} \partial_{\mu}^{\mathrm{LAT}} f\right)(x)+O(a)$.
}

TABLE II. The $\beta, \kappa_{q}^{S}$ and $c_{s w}$ values ${ }^{\mathrm{a}}$ and the lattice volume $V \equiv N_{S}^{3} \times N_{T}$. The collaboration that generated the configurations is given in the last column.

\begin{tabular}{llccc}
\hline \hline$\beta$ & \multicolumn{1}{c}{$\kappa_{q}^{S}$} & $c_{s w}$ & $V$ & Group \\
\hline 5.20 & 0.1342 & 2.0171 & $16^{3} \times 32$ & QCDSF \\
5.20 & 0.1350 & 2.0171 & $16^{3} \times 32$ & UKQCD \\
5.20 & 0.1355 & 2.0171 & $16^{3} \times 32$ & UKQCD \\
5.25 & 0.1346 & 1.9603 & $16^{3} \times 32$ & QCDSF \\
5.25 & 0.1352 & 1.9603 & $16^{3} \times 32$ & UKQCD \\
5.25 & 0.13575 & 1.9603 & $24^{3} \times 48$ & QCDSF \\
5.29 & 0.1340 & 1.9192 & $16^{3} \times 32$ & UKQCD \\
5.29 & 0.1350 & 1.9192 & $16^{3} \times 32$ & QCDSF \\
5.29 & 0.1355 & 1.9192 & $24^{3} \times 48$ & QCDSF \\
5.29 & 0.1359 & 1.9192 & $24^{3} \times 48$ & QCDSF \\
5.40 & 0.1350 & 1.8228 & $24^{3} \times 48$ & QCDSF \\
5.40 & 0.1356 & 1.8228 & $24^{3} \times 48$ & QCDSF \\
5.40 & 0.1361 & 1.8228 & $24^{3} \times 48$ & QCDSF \\
\hline \hline
\end{tabular}

${ }^{\mathrm{a}}$ For the number of trajectories generated for each $\kappa_{q}^{S}$, see for example [42]

$$
\mathcal{A}_{\mu}^{\mathcal{R}}=Z_{A} \mathcal{A}_{\mu}, \quad \mathcal{P}^{\mathcal{S}}(M)=Z_{P}^{\mathcal{S}}(M) \mathcal{P},
$$

giving

$$
m_{q}^{\mathcal{S}}(M)=Z_{\tilde{m}}^{\mathcal{S}}(M) \tilde{m}_{q}, \quad Z_{\tilde{m}}^{\mathcal{S}}(M)=\frac{Z_{A}}{Z_{P}^{\mathcal{S}}(M)},
$$

or in RGI form

$$
m_{q}^{\mathrm{RGI}}=Z_{\tilde{m}}^{\mathrm{RGI}} \tilde{m}_{q}, \quad Z_{\tilde{m}}^{\mathrm{RGI}}=\Delta Z_{m}^{\mathcal{S}}(M) Z_{\tilde{m}}^{\mathcal{S}}(M) .
$$

\section{B. Nonperturbative renormalization}

We shall employ here the RI'-MOM scheme [44], which is easily transcribed to the lattice. Our implementation of this method is described in [45]. As discussed in Sec. II to obtain the RGI quark mass, we must determine both $\Delta Z_{m}^{\mathrm{RI}^{\prime}-\mathrm{MOM}}$ and $Z_{\tilde{m}}^{\mathrm{RI}^{\prime}-\mathrm{MOM}}$. The RGI quark mass can then be easily converted back to the $\overline{\mathrm{MS}}$ scheme.

\section{1. $\Delta Z_{m}^{\mathrm{RI}} \mathrm{I}^{\prime}-\mathrm{MOM}$}

We start with $\Delta Z_{m}^{\mathrm{RI}^{\prime}-\mathrm{MOM}}$. To write down the perturbative expansion, a definition of the coupling constant is required. The anomalous dimension coefficients have been determined to fourth order in $[44,46,47]$ by taking the coupling constant to be $g^{\overline{\mathrm{MS}}}$. Thus the anomalous dimension function is considered as a function of $g^{\overline{\mathrm{MS}}}$. (Other definitions of the coupling constant are possible, more closely related to MOM schemes [48].) So we write

$$
\gamma_{m}^{\mathrm{RI}-\mathrm{MOM}}\left(g^{\overline{\mathrm{MS}}}\right)=d_{m 0}\left(g^{\overline{\mathrm{MS}}}\right)^{2}+d_{m 1}^{\mathrm{RI}-\mathrm{MOM}}\left(g^{\overline{\mathrm{MS}}}\right)^{4}+\ldots
$$

with coefficients given by [47] 


$$
\begin{aligned}
d_{m 1}^{\mathrm{RI}^{\prime}-\mathrm{MOM}}= & -\frac{2}{(4 \pi)^{4}}\left[126-\frac{52}{9} n_{f}\right], \quad d_{m 2}^{\mathrm{RI}^{\prime}-\mathrm{MOM}}=-\frac{2}{(4 \pi)^{6}}\left[\frac{20174}{3}-\frac{3344}{3} \zeta_{3}-\left(\frac{17588}{27}-\frac{128}{9} \zeta_{3}\right) n_{f}+\frac{856}{81} n_{f}^{2}\right], \\
d_{m 3}^{\mathrm{RI}^{\prime}-\mathrm{MOM}}= & -\frac{2}{(4 \pi)^{8}}\left[\frac{141825253}{324}-\frac{7230017}{54} \zeta_{3}+\frac{6160}{3} \zeta_{5}-\left(\frac{3519059}{54}-\frac{298241}{27} \zeta_{3}-\frac{4160}{3} \zeta_{5}\right) n_{f}\right. \\
& \left.+\left(\frac{611152}{243}-\frac{5984}{27} \zeta_{3}\right) n_{f}^{2}-\frac{16024}{729} n_{f}^{3}\right],
\end{aligned}
$$

which allows $\left[\Delta Z_{m}^{\mathrm{RI}}-\operatorname{MOM}\left(\mu_{p}\right)\right]^{-1}$, where $\mu_{p}$ is taken to be the momentum scale in this scheme, to be computed in the usual way,

$$
\left[\Delta Z_{m}^{\mathrm{RI}-\mathrm{MOM}}\left(\mu_{p}\right)\right]^{-1}=\left[2 b_{0} g^{\overline{\mathrm{MS}}}\left(\mu_{p}\right)^{2}\right]^{-d_{m 0} / 2 b_{0}} \exp \left\{\int_{0}^{g^{\overline{\mathrm{MS}}}\left(\mu_{p}\right)} d \xi\left[\frac{\gamma_{m}^{\mathrm{RI}^{\prime}-\mathrm{MOM}}(\xi)}{\beta^{\overline{\mathrm{MS}}}(\xi)}+\frac{d_{m 0}}{b_{0} \xi}\right]\right\}
$$

[This result may also be shown by changing the integration variable in Eq. (9) from some defined $g^{\mathrm{RI}^{\prime}-\mathrm{MOM}}$ to $g^{\mathrm{MS}}$, by using Eq. (10), i.e. $g^{\mathrm{RI}^{\prime}-\mathrm{MOM}}\left(\mu_{p}\right)=G\left(g^{\overline{\mathrm{MS}}}\left(\mu_{p}\right)\right)$.]

In Fig. 2 we show $\left[\Delta Z_{m}^{\mathrm{RI}^{\prime}-\mathrm{MOM}}\right]^{-1}$ as a function of $\mu_{p} / \Lambda^{\overline{\mathrm{MS}}}$. The convergence for $\Delta Z_{m}^{\mathrm{RI}-\mathrm{MOM}}$ seems slightly worse in the region of interest than that for the corresponding $\Delta Z_{m}^{\overline{\mathrm{MS}}}$ as there is more of a change from the 2-loop to 3loop result (see Fig. 1 for a comparison with $\Delta Z_{m}^{\overline{\mathrm{MS}}}$ ). However this is mitigated by the extraction of $\mathrm{RI}^{\prime}$-MOM being performed at a range of scales including higher scales than $2 \mathrm{GeV}$. Thus, for example, a typical value of $\left(a \mu_{p}\right)^{2} \approx 4$ (see Fig. 4) corresponds to $\mu_{p} / \Lambda^{\overline{\mathrm{MS}}} \geq 20$ where the convergence (between the 3-loop and 4-loop results) appears to be better.

\section{2. $Z_{\tilde{m}}^{\mathrm{RI}-\mathrm{MOM}}$}

The RI'-MOM scheme considers amputated Green's functions (practically in the Landau gauge) with an appropriate operator insertion, here either $A$ or $P$. The renormalization point is fixed at some momentum scale $p^{2}=\mu_{p}^{2}$,

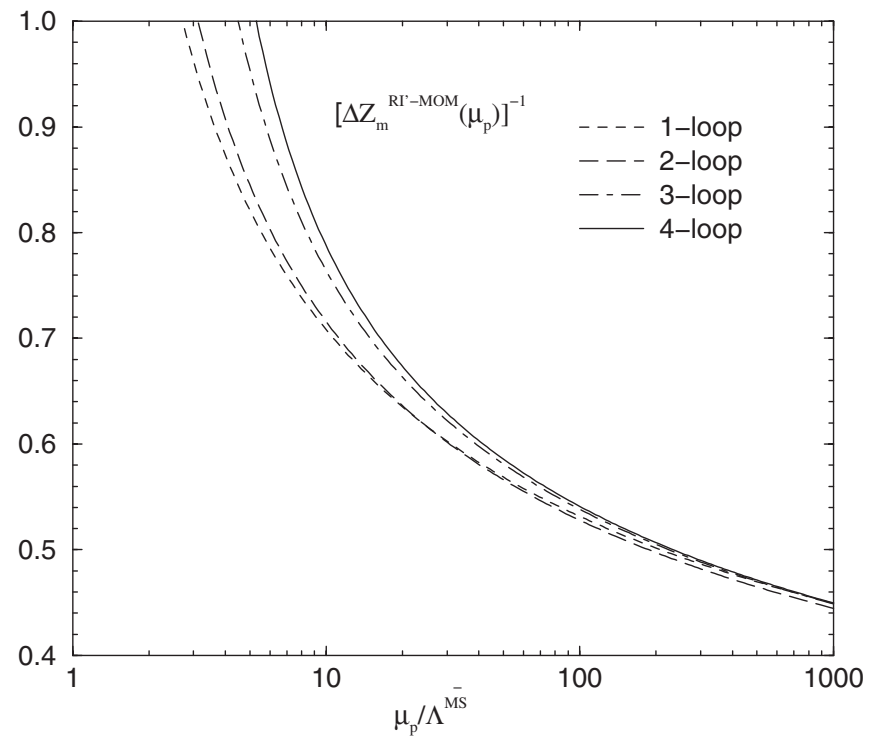

FIG. 2. $\left[\Delta Z_{m}^{\mathrm{RI}^{\prime}-\mathrm{MOM}}\left(\mu_{p}\right)\right]^{-1}$ versus $\mu_{p} / \Lambda^{\overline{\mathrm{MS}}}$. and thus we have, e.g. [44,45],

$$
Z_{O}^{\mathrm{RI}^{\prime}-\operatorname{MOM}}\left(\mu_{p}\right)=\left.\frac{Z_{q}^{\mathrm{RI}-\operatorname{MOM}}(p)}{\frac{1}{12} \operatorname{tr}\left[\Gamma_{O}(p) \Gamma_{O, \text { BORN }}^{-1}(p)\right]}\right|_{p^{2}=\mu_{p}^{2}}
$$

where $\Gamma_{O}$ are one-particle irreducible (1PI) vertex functions, and $Z_{q}$ is the wave-function renormalization. We have generated $\Gamma_{O}$ only at values of the sea quark mass, i.e. the values in Table II.

To obtain $Z_{\tilde{m}}^{\mathrm{RI}-\mathrm{MOM}}$ we need both $Z_{A}$ and $Z_{P}^{\mathrm{RI}}-\mathrm{MOM}$ in the chiral limit. $Z_{A}$ is unproblematical, we make a linear fit of the form

$$
Z_{A}=A_{A}+B_{A} a m_{q},
$$

where $a m_{q}$ is defined in Eq. (38) and $\kappa_{q c}^{S}$ has been determined in [11].

From the Ward identity obeyed by $\Gamma_{P}$, we expect, due to chiral symmetry breaking, that $\left(Z_{P}^{\mathrm{RI}^{\prime}-\mathrm{MOM}}\right)^{-1}$ develops a pole in the quark mass. Hence we have $Z_{P} \rightarrow 0$ with the quark mass. Thus following [49] we try a fit of the form ${ }^{7}$

$$
\left(Z_{P}^{\mathrm{RI}^{\prime}-\mathrm{MOM}}\right)^{-1}=A_{P}+\frac{B_{P}}{a m_{q}} .
$$

From the operator product expansion $[50,51]$ we expect

$$
B_{P}\left(\mu_{p}\right) \propto \frac{1}{\left(a \mu_{p}\right)^{2}},
$$

where the constant of proportionality is proportional to the chiral condensate. Thus we see that as the scale increases, the $B_{P}$ coefficient decreases.

A chiral extrapolation using Eqs. (47) and (48) has been made using only the sea data sets to determine the functions $A_{A}\left(\mu_{p}\right)$ and $A_{P}\left(\mu_{p}\right)$, respectively. The lattice momenta originally chosen for the heaviest quark masses were kept fixed for the extrapolation over the different masses. If another data set did not have a particular momentum a linear interpolation was performed between the adjacent momenta straddling the given momentum.

\footnotetext{
${ }^{7}$ It made little difference to the $A_{P}$ (or $A_{A}$ ) coefficients whether $a \tilde{m}_{q}$ or $\left(a m_{p s}\right)^{2}$ is used.
} 
M. GÖCKELER et al.
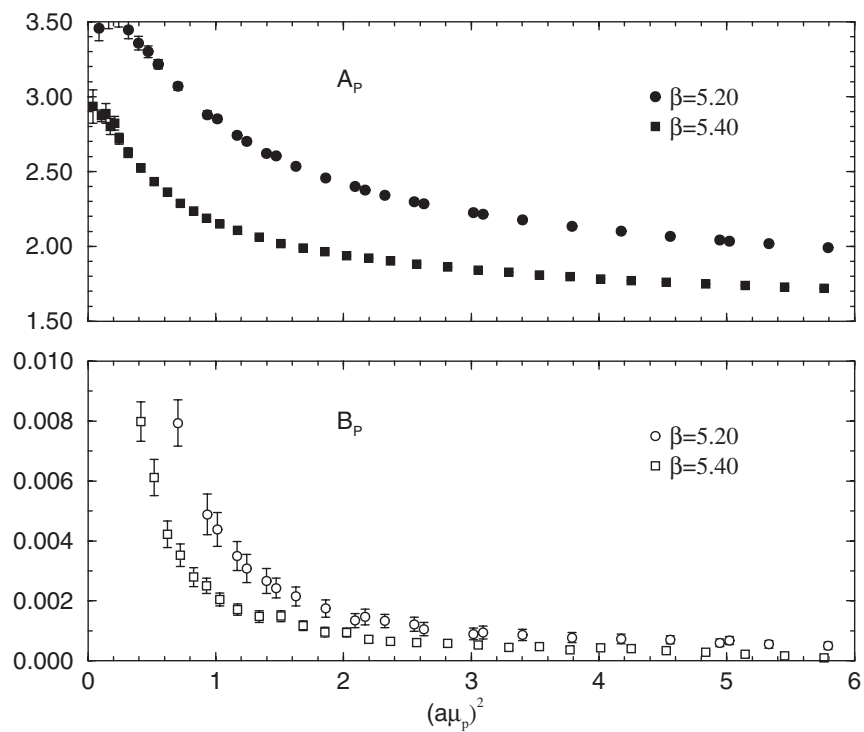

FIG. 3. $A_{P}$ and $B_{P}$ for $Z_{P}^{\mathrm{RI}}-\mathrm{MOM}$ for $\beta=5.20$ and $\beta=5.40$.

Results for $Z_{P}^{\mathrm{RI}}{ }^{\prime}-\mathrm{MOM}$ are shown for $\beta=5.20,5.40$ in Fig. 3. $B_{P}$ is small and decreasing with increasing $\left(a \mu_{p}\right)^{2}$ as required from Eq. (49). [Note that numerically, there is evidence for a term of the form $B_{P} / a m_{q}$ as attempting a linear extrapolation, as for $Z_{A}$ in Eq. (47), gave a substantial increase in the fit $\chi^{2}$.] Although we do not determine the chiral condensate [52] in this section (see Sec. VIA), we note that numerically the Goldstone pion contamination to $\Gamma_{P}$ appears to be small [53].

\section{3. $Z_{\tilde{m}}^{\mathrm{RGI}}$}

Taking these values for $A_{A} \equiv Z_{A}$ and $A_{P} \equiv Z_{P}^{\mathrm{RI}}{ }^{\prime}-\mathrm{MOM}$, forming the ratio $Z_{A} / Z_{P}^{\mathrm{RI}-\mathrm{MOM}}$ and multiplying by

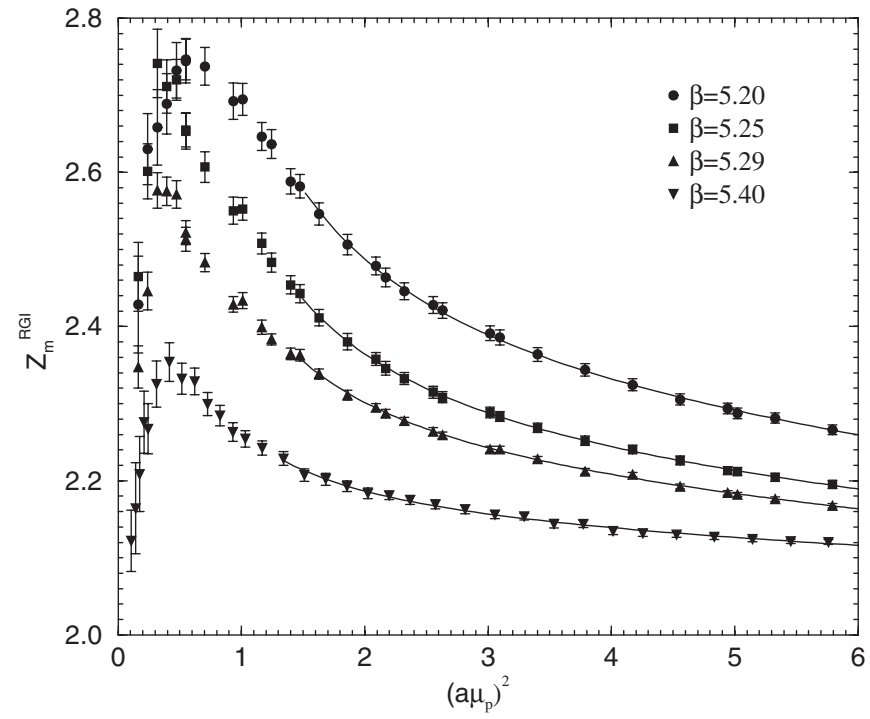

FIG. 4. $Z_{\tilde{m}}^{\mathrm{RGI}}$ for $\beta=5.20$ (filled circles), $\beta=5.25$ (filled squares), $\beta=5.29$ (filled upper triangles), $\beta=5.40$ (filled lower triangles) together with fits as in Eq. (50).
PHYSICAL REVIEW D 73, 054508 (2006)

TABLE III. Values of $Z_{\tilde{m}}^{\text {RGI }}$ from the NP method of Sec. V B 3.

\begin{tabular}{lc}
\hline \hline$\beta$ & $Z_{\tilde{m}}^{\text {RGI }}$ \\
\hline 5.20 & $2.270(12)$ \\
5.25 & $2.191(24)$ \\
5.29 & $2.177(14)$ \\
5.40 & $2.124(06)$ \\
\hline \hline
\end{tabular}

$\Delta Z_{m}^{\mathrm{RI}-\mathrm{MOM}}$ will then give $Z_{\tilde{m}}^{\mathrm{RGI}}$. This should be independent of $\left(a \mu_{p}\right)^{2}$. Some results are shown in Fig. 4. Because of cutoff effects, nonperturbative contributions etc., they are not quite constant although the curves become flatter for increasing $\beta$. To allow for the nonconstant remnants we make a phenomenological fit of the form

$$
F\left(a \mu_{p}\right)=r_{1}+r_{2}\left(a \mu_{p}\right)^{2}+\frac{r_{3}}{\left(a \mu_{p}\right)^{2}},
$$

where we associate $Z_{\tilde{m}}^{\mathrm{RGI}}$ with $r_{1}$. This gives the results in Table III. We start the fit range at $\left(a \mu_{p}\right)^{2}=1.5$, the fit results for $r_{1}$ were found to be insensitive to decreasing the fit range.

\section{Comparison of $Z_{\tilde{m}}^{\mathrm{RGI}}$ with other results}

As many computations of the strange quark mass have used tadpole-improved perturbation theory together with a boosted coupling constant for the determination of the renormalization constant, it is of interest to compare our results obtained in the previous section with this approach. Our variation of this method, tadpole-improved renormalization-group-improved boosted perturbation theory or TRB-PT, is described in $[43,54]$. Here we recapitulate the method. Regarding the lattice as a "scheme," then from Eq. (8) we can write

$$
m_{q}^{\mathrm{RGI}}=\Delta Z_{\tilde{m}}^{\mathrm{LAT}}(a) \tilde{m}_{q}(a)
$$

where the renormalization-group-improved $\Delta Z_{\tilde{m}}^{\mathrm{LAT}}(a)$ is given by Eq. (9). Furthermore, in this "lattice" scheme we choose to use $g_{\square}^{2}=g_{0}^{2} / u_{0 c}^{4}$ where $u_{0}^{4}=\left\langle\frac{1}{3} \operatorname{Tr} U^{\square}\right\rangle\left(U^{\square}\right.$ being the product of links around an elementary plaquette) rather than $g_{0}$, as series expansions in $g_{\square}$ are believed to have better convergence. This is boosted perturbation theory. (We shall use chirally extrapolated plaquette values as determined in [19] at our $\beta$ values and so we add a subscript " $c$ " to $u_{0}$.) In the tadpole-improved, or mean field approximation, renormalization constants for operators with no derivatives are $\propto u_{0 c}$, which indicates that $Z_{\tilde{m}}^{\mathrm{RGI}} u_{0 c}^{-1}$ will converge faster than $Z_{\tilde{m}}^{\mathrm{RGI}}$ alone so we rewrite the two-loop equation Eq. (11) as ${ }^{8}$

\footnotetext{
${ }^{8}$ The TRB-PT subscript in brackets is there only to distinguish the results from those obtained in Sec. VB.
} 


$$
\begin{aligned}
Z_{\tilde{m}}^{\mathrm{RGI}(\mathrm{TRB}-\mathrm{PT})} \equiv & \Delta Z_{\tilde{m}}^{\mathrm{LAT}}(a) \\
= & u_{0 c}\left[2 b_{0} g_{\square}^{2}\right]^{d_{m 0} / 2 b_{0}} \\
& \times\left[1+\frac{b_{1}}{b_{0}} g_{\square}^{2}\right]^{\left(b_{0} d_{\tilde{m} 1}^{\mathrm{LAT}}-b_{1} d_{m 0}\right) / 2 b_{0} b_{1}+\left(p_{1} / 4\right)\left(b_{0} / b_{1}\right)},
\end{aligned}
$$

where $p_{1}$ is the first coefficient in the expansion of $u_{0 c}$, i.e. $u_{0 c}=1-\frac{1}{4} g_{0}^{2} p_{1}+\ldots$ with $p_{1}=\frac{1}{3}$.

It remains to determine $d_{\tilde{m} 1}^{\mathrm{LAT}}$. This may be found by relating the (known) perturbative result for $Z_{\tilde{m}}^{\overline{\mathrm{MS}}}$ to $\Delta Z_{\tilde{m}}^{\mathrm{LAT}}$ (for simplicity at the scale $\mu=1 / a$ ) by

$$
Z_{\tilde{m}}^{\overline{\mathrm{MS}}}(1 / a)=\frac{\Delta Z_{\tilde{m}}^{\mathrm{LAT}}(a)}{\Delta Z_{m}^{\overline{\mathrm{MS}}}(1 / a)}=1-g_{0}^{2} B_{\tilde{m}}^{\overline{\mathrm{MS}}}(1)+\ldots,
$$

where

$$
\begin{aligned}
B_{\tilde{m}}^{\overline{\mathrm{MS}}}\left(c_{s w}\right)= & \frac{4 / 3}{(4 \pi)^{2}}\left(-6.79916+2.4967 c_{s w}\right. \\
& \left.-4.28739 c_{s w}^{2}\right) .
\end{aligned}
$$

This result for $B_{\tilde{m}}^{\overline{\mathrm{MS}}} \equiv B_{A}-B_{P}^{\overline{\mathrm{MS}}}$ is taken from [55]. (Indeed we could alternatively consider TRB-PT for $Z_{A}$ and $Z_{P}^{\overline{\mathrm{MS}}}$ separately and then form the ratio. The results turned out to be about $1 \%$ lower than those presented here.)

Expanding the ratio in Eq. (53), by using the results in Eqs. (52) and (11) for the "lattice" and $\overline{\mathrm{MS}}$ schemes, respectively, and $t_{1}$, defined in Eq. (10) (which using the notation of [19] is numerically given by $t_{1}=t_{1}^{\mathrm{LAT}}(1)$, with $t_{1}^{\mathrm{LAT}}\left(c_{s w}\right)=0.4682013-n_{f}(0.0066960-$ $\left.\left.0.0050467 c_{s w}+0.0298435 c_{s w}^{2}\right)\right)$ gives finally

$$
\begin{aligned}
d_{\tilde{m} 1}^{\mathrm{TRB}-\mathrm{PT}} & =d_{m 1}^{\overline{\mathrm{MS}}}+d_{m 0}\left(t_{1}-p_{1}\right)-2 b_{0} B_{\tilde{m}}^{\overline{\mathrm{MS}}}(1) \\
& \equiv-\frac{4.04873}{(4 \pi)^{4}} .
\end{aligned}
$$

Thus from Eq. (52) various values of $\Delta Z_{\tilde{m}}^{\mathrm{LAT}}$, or equivalently $Z_{\tilde{m}}^{\mathrm{RGI}(\mathrm{TRB}-\mathrm{PT})}$, can be found. Results are given in Table IV.

We now turn to a comparison of the results. In Fig. 5 we plot $Z_{\tilde{m}}^{\mathrm{RGI}}$ versus $\beta$. Our NP results from Sec. VB are shown as filled circles. They are to be compared with the TRB-PT results denoted by empty squares. While there is a difference between the results, it is decreasing for $\beta \rightarrow \infty$ and thus may be primarily due to remnant $O\left(a^{2}\right)$ effects,

TABLE IV. Values of $Z_{\tilde{m}}^{\mathrm{RGI}(\mathrm{TRB}-\mathrm{PT})}$ from Sec. VC.

\begin{tabular}{lc}
\hline \hline$\beta$ & $Z_{\tilde{m}}^{\text {RGI(TRB-PT) }}$ \\
\hline 5.20 & 1.837 \\
5.25 & 1.851 \\
5.29 & 1.862 \\
5.40 & 1.891 \\
\hline \hline
\end{tabular}

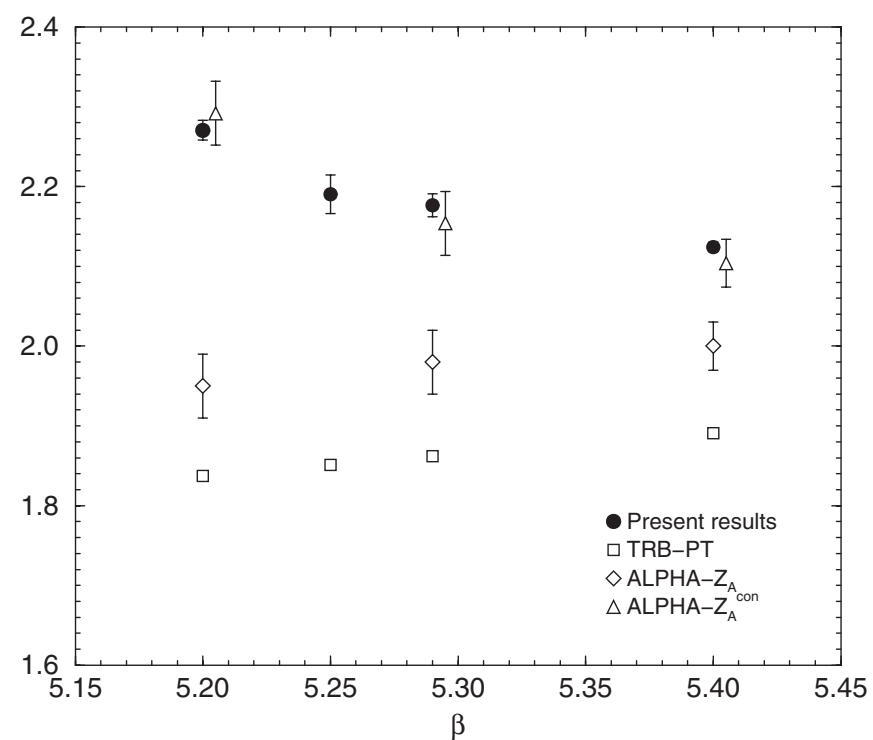

FIG. 5. $Z_{\tilde{m}}^{\mathrm{RGI}}$ versus $\beta$. The black circles are the results from Table III, while the open squares are the TRG-PT results from Table IV. Furthermore, the open diamonds and triangles are the NP results from [12], using the two different results for the axial renormalization constant [56]. (The empty triangle results have been slightly displaced for clarity.)

which disappear in the continuum limit. That various determinations of $Z_{\tilde{m}}^{\text {RGI }}$ have different numerical values can be seen from the results of [12] (open diamonds and triangles). In these results two different definitions of the axial renormalization constant have been used [56]. $Z_{A}$ is computed when dropping certain disconnected diagrams, while $Z_{A}^{\mathrm{CON}}$ includes them. The difference between the two definitions is an $O\left(a^{2}\right)$ effect. Using $Z_{A}^{\mathrm{CON}}$ in $Z_{\tilde{m}}^{\mathrm{RGI}}$ leads, perhaps coincidently, to very similar results to our NP results.

Investigating the possibility of $O\left(a^{2}\right)$ differences a little further, we note that if we have two definitions of $Z_{\tilde{m}}^{\text {RGI }}$ then if both are equally valid, forming the ratio should yield

$$
R_{\tilde{m}}^{X} \equiv \frac{Z_{\tilde{m}}^{\mathrm{RGI}(X)}}{Z_{\tilde{m}}^{\mathrm{RGI}}}=1+O\left(a^{2}\right)
$$

where $Z_{\tilde{m}}^{\mathrm{RGI}}$ is the result of Sec. VB and $X$ is some alternative definition (i.e. TRB-PT, ALPHA- $Z_{A}$, ALPHA- $Z_{A}^{\mathrm{CON}}$ ). In Fig. 6 we plot this ratio for these alternative definitions. The $r_{0} / a$ values used for the $x$-axis are found by extrapolating the $r_{0} / a$ results to the chiral limit. This extrapolation and results for $\left(r_{0} / a\right)_{c}$ are given in [19].

We see that (roughly) all three ratios extrapolate to 1 which implies that any of the four determinations of $Z_{\tilde{m}}^{\mathrm{RGI}}$ may be used. This includes the TRB-PT result. Of course other TI determinations might not have this property, and also their validity always has to be checked against a NP determination, so this result here is of limited use; it is always essential to make a NP determination of the renormalization constant. Furthermore, it is also to be noted that 
M. GÖCKELER et al.

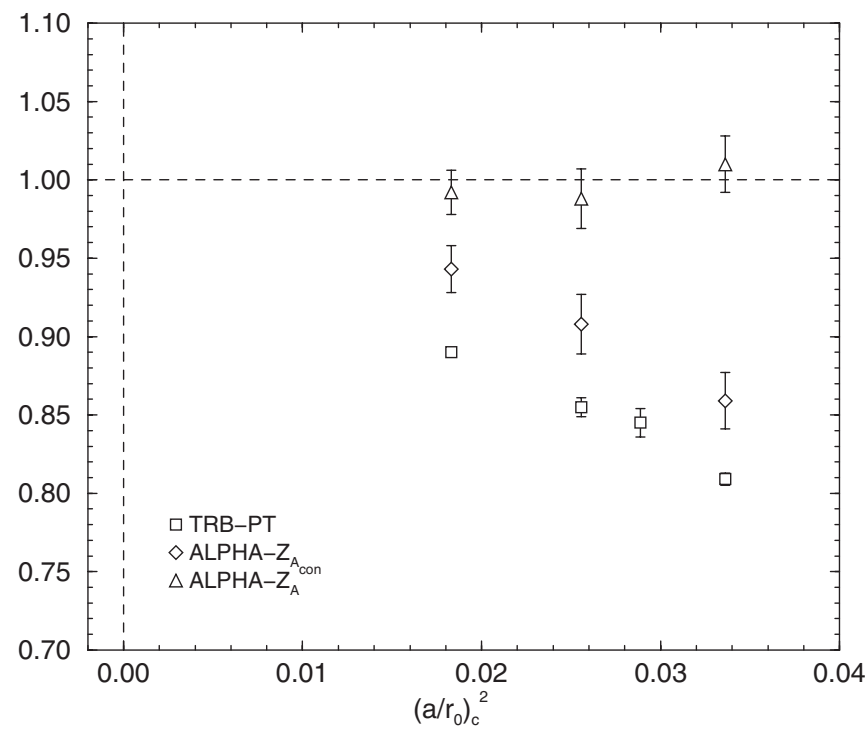

FIG. 6. $R_{\tilde{m}}^{X}$ versus $\left(a / r_{0}\right)_{c}^{2}$ for $X=$ TRB - PT (open squares), $X=$ ALPHA $-Z_{A}$ (open diamonds) and $X=$ ALPHA $-Z_{A}^{\mathrm{CON}}$ (open triangles).

different determinations can have rather different $O\left(a^{2}\right)$ corrections, so a continuum extrapolation is always necessary.

\section{RESULTS}

\section{A. Generalities}

In Figs. 7-10 we plot the ratio $r_{0} m_{q}^{\mathrm{RGI}} /\left(r_{0} m_{p s}\right)^{2}$ against $\left(r_{0} m_{p s}\right)^{2}$, where $r_{0} \equiv r_{0}^{S}$ (i.e. $r_{0}$ depends only on the sea quark mass, $S$ ). The numerical values of $r_{0} / a$ are given in
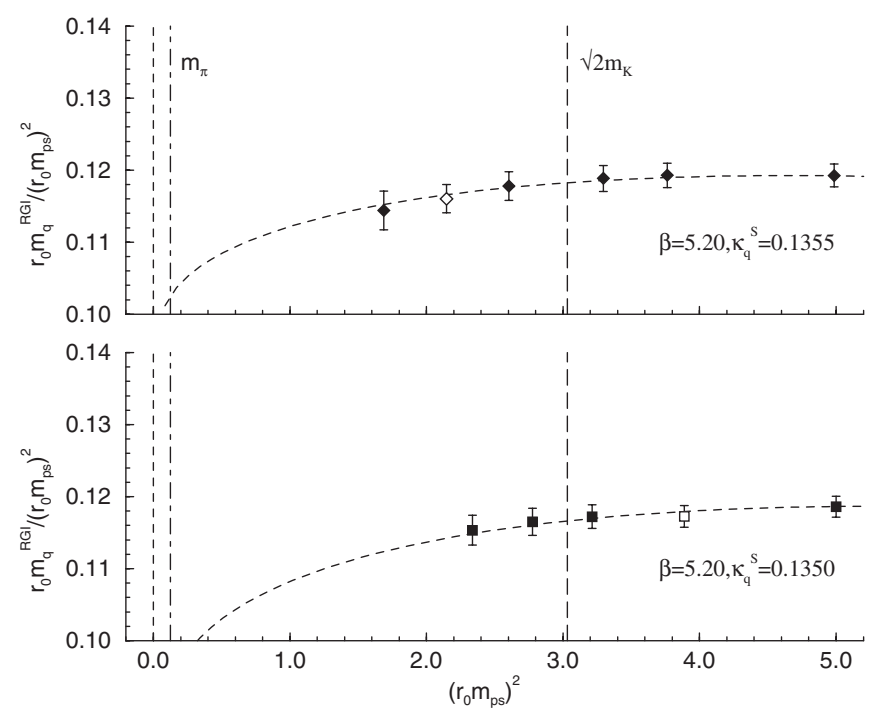

FIG. 7. The ratio $r_{0} m_{q}^{\mathrm{RGI}} /\left(r_{0} m_{p s}\right)^{2}$ against $\left(r_{0} m_{p s}\right)^{2}$ for $\beta=$ 5.20. The fit is described in the text. Results for equal sea and valence quark masses are denoted by an open symbol; partially quenched results by filled symbols. The labeled dashed and dashed-dotted lines are also explained in the text.
PHYSICAL REVIEW D 73, 054508 (2006)
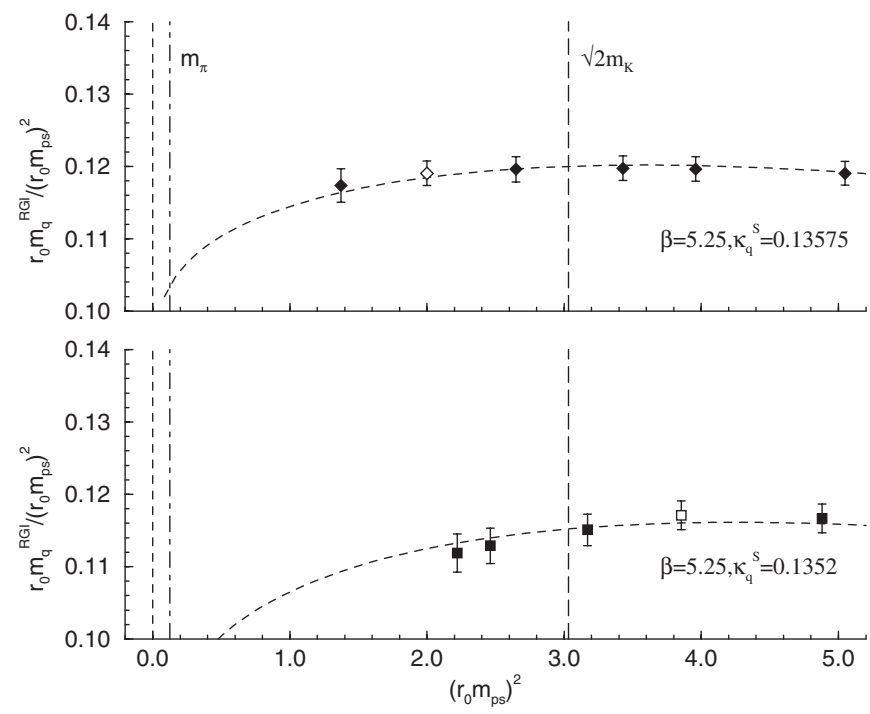

FIG. 8. The ratio $r_{0} m_{q}^{\mathrm{RGI}} /\left(r_{0} m_{p s}\right)^{2}$ against $\left(r_{0} m_{p s}\right)^{2}$ for $\beta=$ 5.25. Notation as for Fig. 7.

[19], Table II. $r_{0}$ seems to be a good scale to use because this ratio numerically does not vary much, as can be seen from the figures.

Using Eq. (25) (the case of degenerate valence quarks) we set

$$
y_{V}=r_{0} m_{q}^{\mathrm{RGI}}, \quad M_{p s}^{S}=r_{0} m_{p s}^{S}, \quad M_{p s}^{V}=r_{0} m_{p s},
$$

to give a fit equation of the form

$$
\begin{aligned}
\frac{r_{0} m_{q}^{\mathrm{RGI}}}{\left(r_{0} m_{p s}\right)^{2}}= & c_{a}^{\mathrm{RGI}}+c_{b}^{\mathrm{RGI}}\left(r_{0} m_{p s}^{S}\right)^{2}+c_{c}^{\mathrm{RGI}}\left(r_{0} m_{p s}\right)^{2} \\
& +c_{d}^{\mathrm{RGI}}\left(\left(r_{0} m_{p s}^{S}\right)^{2}-2\left(r_{0} m_{p s}\right)^{2}\right) \ln \left(r_{0} m_{p s}\right)^{2} .
\end{aligned}
$$

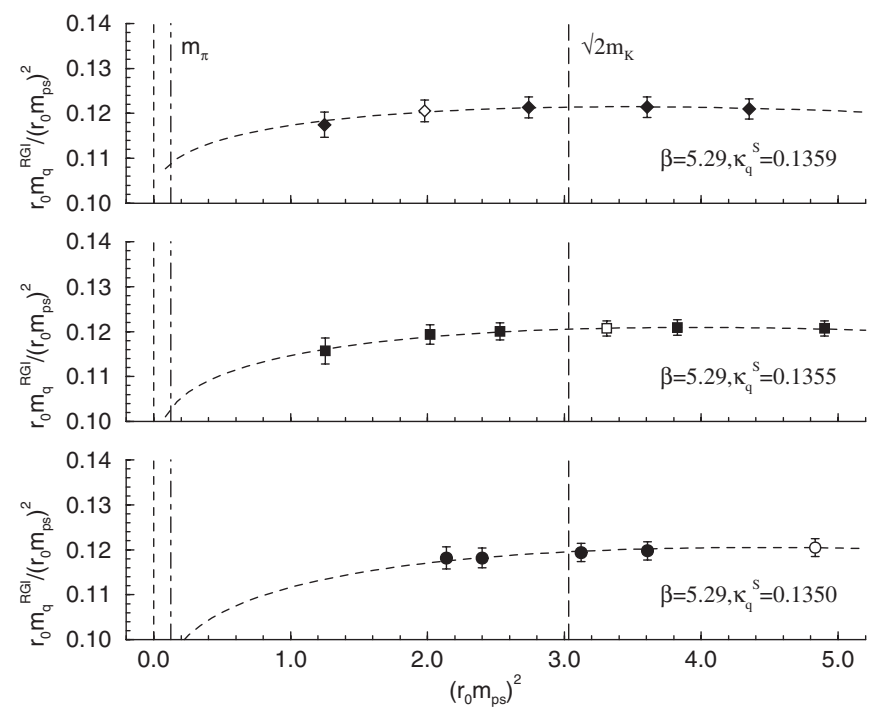

FIG. 9. The ratio $r_{0} m_{q}^{\mathrm{RGI}} /\left(r_{0} m_{p s}\right)^{2}$ against $\left(r_{0} m_{p s}\right)^{2}$ for $\beta=$ 5.29. Notation as for Fig. 7. 


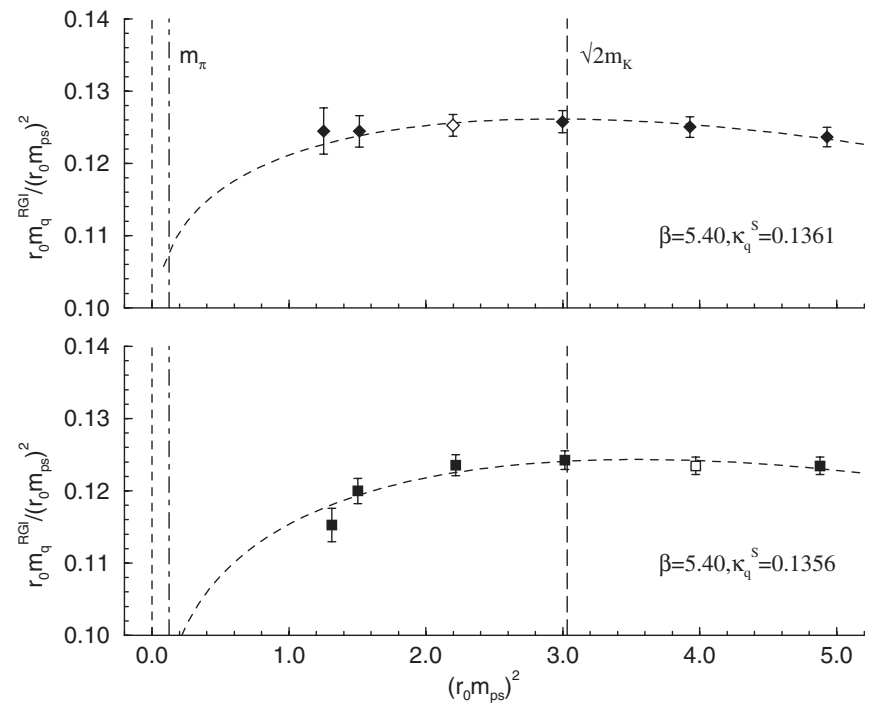

FIG. 10. The ratio $r_{0} m_{q}^{\mathrm{RGI}} /\left(r_{0} m_{p s}\right)^{2}$ against $\left(r_{0} m_{p s}\right)^{2}$ for $\beta=$ 5.40. Notation as for Fig. 7.

We use this equation to determine the coefficients $c_{a}^{\text {RGI }}$ and $c_{i}^{\mathrm{RGI}}, i=b, c, d$. The coefficients depend only on lattice simulation quantities and the unit chosen, and not on the scale as given, for example, in Eq. (15) or Eq. (16). This can be useful, as an aid, when making comparisons with other results.

As already mentioned, up to this order in chiral perturbation theory no input from lattice simulations with nondegenerate valence quark masses is needed. To test also numerically that the effects from nondegenerate quark masses are indeed small, we calculated correlation functions using nondegenerate valence quark masses $m_{q}^{A} \neq m_{q}^{B}$ as well as degenerate valence quark masses with $m_{q}^{S} \equiv$ $\left(m_{q}^{A}+m_{q}^{B}\right) / 2$. We found the relevant quantities $a m_{p s}$ and $a \tilde{m}_{q}$ to differ by $\lesssim 1 \%$.

In Figs. $7-10$ the dashed lines (labeled " $\sqrt{2} m_{K}$ ") represent a fictitious particle composed of two strange quarks, which at LO $\chi \mathrm{PT}$ gives from Eq. (29) [or equivalently Eq. (63)] the line $\left(r_{0} m_{p s}\right)^{2} \equiv\left(r_{0} m_{K^{+}}\right)^{2}+\left(r_{0} m_{K^{0}}\right)^{2}-$ $\left(r_{0} m_{\pi^{+}}\right)^{2}$, while the dashed-dotted lines (labeled " $m_{\pi}$ ") represent a fictitious pion with mass degenerate $u$ and $d$ quarks given from Eq. (30) [or equivalently Eq. (64)] by $\left(r_{0} m_{\pi^{+}}\right)^{2}$.

The presence of a chiral logarithm in the data manifests itself in the bending of the results for smaller quark mass, which can be seen in Figs. 7-10. Results for the fit parameters are given in Table $\mathrm{V}$.

Numerically we expect the leading order in $\chi \mathrm{PT}$ to be dominant with the NLO giving only minor corrections, ${ }^{9}$ i.e.

\footnotetext{
${ }^{9}$ Alternative plots, using constant $a$ so $a \tilde{m}_{q} /\left(a m_{p s}\right)^{2}$ against $\left(a m_{p s}\right)^{2}$ or equivalently $r_{0} \tilde{m}_{q} /\left(r_{0} m_{p s}\right)^{2}$ against $\left(r_{0} m_{p s}\right)^{2}$ using $\left(r_{0} / a\right)_{c}$ which is the chirally extrapolated $r_{0} / a$ would give larger NLO corrections.
}

TABLE V. Values of $c_{a}^{\mathrm{RGI}}$ and $c_{i}^{\mathrm{RGI}}(i=b, c, d)$ together with their extrapolated (continuum) values $(\beta=\infty)$.

\begin{tabular}{lcccc}
\hline \hline$\beta$ & $c_{a}^{\text {RGI }}$ & $c_{b}^{\text {RGI }}$ & $c_{c}^{\text {RGI }}$ & $c_{d}^{\text {RGI }}$ \\
\hline 5.20 & $0.1115(53)$ & $-0.00227(187)$ & $0.00557(491)$ & $0.00121(133)$ \\
5.25 & $0.1169(42)$ & $-0.00428(160)$ & $0.00612(399)$ & $0.00153(111)$ \\
5.29 & $0.1166(29)$ & $-0.00199(117)$ & $0.00470(287)$ & $0.00121(090)$ \\
5.40 & $0.1218(24)$ & $-0.00324(079)$ & $0.00646(179)$ & $0.00189(056)$ \\
$\infty$ & $0.1330(74)$ & $-0.00378(254)$ & $0.00789(607)$ & $0.00275(179)$ \\
\hline \hline
\end{tabular}

we expect $c_{a}^{\mathrm{RGI}} \gg\left(r_{0} m_{p s}\right)^{2} c_{i}^{\mathrm{RGI}}(i=b, c, d)$ and this is indeed found. Using these results for $c_{a}^{\mathrm{RGI}}, c_{i}^{\mathrm{RGI}}(i=$ $b, c, d)$ in Fig. 11 we present their continuum extrapolations. The $r_{0} / a$ values used for the $x$-axis are found by extrapolating the previously used $r_{0} / a$ results to the chiral limit. This extrapolation and results (for $\left.\left(r_{0} / a\right)_{c}\right)$ are also given in [19]. This gives values in the last line of Table $\mathrm{V}$ $(\beta=\infty)$.

As a first check on these results and to find some idea of possible systematic effects, we have varied the fit interval from the chosen $\left(r_{0} m_{p s}\right)^{2} \lesssim 5$ to $\left(r_{0} m_{p s}\right)^{2} \lesssim 4$ or 6 or $\infty$, i.e. include all the data. [We can go no lower than $\left(r_{0} m_{p s}\right)^{2} \lesssim 4$ if we wish to have at least two sea quark masses in the fit for each $\beta$.] There was little change in the fit result and we shall take these changes as a systematic error, see below and Sec. VIB. Using $\left(r_{0} m_{p s}\right)^{2} \lesssim 5$ means that our pseudoscalar masses range from about $440 \mathrm{MeV}$ to about double that value.

As a second check on the validity of these results, let us relate them to the LECs in Eqs. (26)-(28) (in the continuum limit). From Table V and Eq. (28) we find that

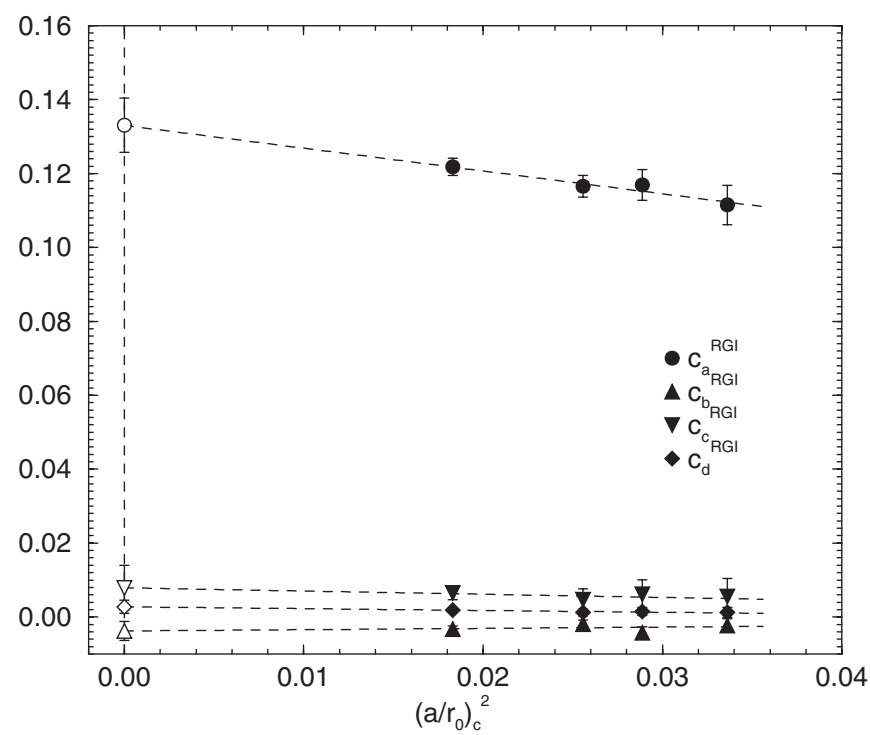

FIG. 11. $c_{a}^{\mathrm{RGI}}$ (filled circles), $c_{b}^{\mathrm{RGI}}$ (filled upper triangles) $c_{c}^{\mathrm{RGI}}$ (filled lower triangles) and $c_{d}^{\text {RGI }}$ (filled diamonds) versus $\left(a / r_{0}\right)_{c}^{2}$. The $\left(r_{0} / a\right)_{c}$ i.e. the chirally extrapolated values for $r_{0} / a$ are used. The open symbols represent the values of $c_{a}^{\mathrm{RGI}}$ and $c_{i}^{\mathrm{RGI}}$ $(i=b, c, d)$ in the continuum limit. 


$$
2 \alpha_{6}-\alpha_{4} \approx-0.21, \quad 2 \alpha_{8}-\alpha_{5} \approx 1.25 .
$$

These numbers are to be compared with the results of Eq. (21) namely $\approx-0.24$ and $\approx 1.02$ respectively. Furthermore, we have from Eqs. (18), (19), (22), and (57) the relations

$$
c_{\chi}=\frac{2 B_{0}^{\mathrm{RGI}}}{\left(4 \pi f_{0}\right)^{2} r_{0}}, \quad c_{m}=\frac{1}{4 \pi f_{0} r_{0}},
$$

where $B_{0}^{\mathrm{RGI}}=\left[\Delta Z_{m}^{\mathcal{S}}(M)\right]^{-1} B_{0}^{\mathcal{S}}$, which give

$$
B_{0}^{\mathrm{RGI}}=\frac{1}{2 r_{0} c_{a}^{\mathrm{RGI}}} .
$$

Together with Eq. (20) (using $f_{0} \approx f_{\pi}$ ) and $\left[\Delta Z_{m}^{\overline{\mathrm{MS}}}(2 \mathrm{GeV})\right]^{-1}$ from Table $\mathrm{I}$ this leads to [cf. Equation (21)],

$$
\begin{aligned}
& \langle\bar{q} q\rangle^{\overline{\mathrm{MS}}}(2 \mathrm{GeV}) \\
& \quad=-\left\{\begin{array}{ll}
(263(5)(5)(5) \mathrm{MeV})^{3} & \text { for } r_{0}=0.5 \mathrm{fm} \\
(267(5)(5)(5) \mathrm{MeV})^{3} & \text { for } r_{0}=0.467 \mathrm{fm}
\end{array},\right.
\end{aligned}
$$

where, as discussed in Sec. II, we consider two scales $r_{0}=$ $0.5 \mathrm{fm}$ and $r_{0}=0.467 \mathrm{fm}$. The first error is statistical and the second is systematic $\approx 5 \mathrm{MeV}$ determined by the change in $c_{a}^{\mathrm{RGI}}$ when changing the fit interval, as discussed above. The third (systematic) error is due to the choice of $r_{0}$ scale, taken here as the difference between the results and also $\approx 5 \mathrm{MeV}$.

We find it encouraging that the $c_{a}^{\mathrm{RGI}}$ and $c_{i}^{\mathrm{RGI}}(i=$ $b, c, d)$ results from the fit give numbers in rough agreement with phenomenological expectations for $2 \alpha_{6}-\alpha_{4}$, $2 \alpha_{8}-\alpha_{5}$ and the chiral condensate (and also with other lattice determinations of the chiral condensate, e.g. $[52,57])$. However, to obtain more accurate results will require much more precise numerical data. ${ }^{10}$

\section{B. The quark masses}

We now turn to the evaluation of the strange (and light) quark masses. After finding the coefficients $c_{a}^{\mathrm{RGI}}, c_{i}^{\mathrm{RGI}}(i=$ $b, c, d$ ), these can be substituted into Eq. (29) to give for the strange quark mass

$$
\begin{aligned}
r_{0} m_{s}^{\mathrm{RGI}}= & c_{a}^{\mathrm{RGI}}\left[\left(r_{0} m_{K^{+}}\right)^{2}+\left(r_{0} m_{K^{0}}\right)^{2}-\left(r_{0} m_{\pi^{+}}\right)^{2}\right]+\left(c_{b}^{\mathrm{RGI}}-c_{d}^{\mathrm{RGI}}\right)\left[\left(r_{0} m_{K^{+}}\right)^{2}+\left(r_{0} m_{K^{0}}\right)^{2}\right]\left(r_{0} m_{\pi^{+}}\right)^{2} \\
& +\frac{1}{2}\left(c_{c}^{\mathrm{RGI}}+c_{d}^{\mathrm{RGI}}\right)\left[\left(r_{0} m_{K^{+}}\right)^{2}+\left(r_{0} m_{K^{0}}\right)^{2}\right]^{2}-\left(c_{b}^{\mathrm{RGI}}+c_{c}^{\mathrm{RGI}}\right)\left(r_{0} m_{\pi^{+}}\right)^{4}-c_{d}^{\mathrm{RGI}}\left[\left(r_{0} m_{K^{+}}\right)^{2}+\left(r_{0} m_{K^{0}}\right)^{2}\right]\left[\left(r_{0} m_{K^{+}}\right)^{2}\right. \\
& \left.+\left(r_{0} m_{K^{0}}\right)^{2}-\left(r_{0} m_{\pi^{+}}\right)^{2}\right] \ln \left(\left(r_{0} m_{K^{+}}\right)^{2}+\left(r_{0} m_{K^{0}}\right)^{2}-\left(r_{0} m_{\pi^{+}}\right)^{2}\right)+c_{d}^{\mathrm{RGI}}\left(r_{0} m_{\pi^{+}}\right)^{4} \ln \left(r_{0} m_{\pi^{+}}\right)^{2} .
\end{aligned}
$$

Similarly for the light quark mass we have

$$
\begin{aligned}
r_{0} m_{u d}^{\mathrm{RGI}}= & c_{a}^{\mathrm{RGI}}\left(r_{0} m_{\pi^{+}}\right)^{2}+\left(c_{b}^{\mathrm{RGI}}+c_{c}^{\mathrm{RGI}}\right)\left(r_{0} m_{\pi^{+}}\right)^{4} \\
& -c_{d}^{\mathrm{RGI}}\left(r_{0} m_{\pi^{+}}\right)^{4} \ln \left(r_{0} m_{\pi^{+}}\right)^{2} .
\end{aligned}
$$

We first consider the strange quark mass. As can be seen from Table V or Fig. 11, the errors of the NLO parameters, i.e. $c_{i}^{\mathrm{RGI}}(i=b, c, d)$ are the same size as the signal itself and thus using them directly in Eq. (63) simply gives a change in the $\mathrm{LO}$ result (i.e. using only the $c_{a}^{\mathrm{RGI}}$ term) of a few percent, together with a similar increase in the error (especially using error propagation; note that the third and fifth terms in Eq. (63) give the main contribution to the NLO term).

To reduce the total error on the result, it proved advantageous to use Eq. (63) to eliminate $c_{a}^{\mathrm{RGI}}$ from Eq. (58) in terms of

$$
c_{a^{\prime}}^{\mathrm{RGI}} \equiv \frac{r_{0} m_{s}^{\mathrm{RGI}}}{\left(r_{0} m_{K^{+}}\right)^{2}+\left(r_{0} m_{K^{0}}\right)^{2}-\left(r_{0} m_{\pi^{+}}\right)^{2}} .
$$

This results in a modified fit function of the form

$$
\begin{aligned}
\frac{r_{0} m_{q}^{\mathrm{RGI}}}{\left(r_{0} m_{p s}\right)^{2}}= & c_{a^{\prime}}^{\mathrm{RGI}}+c_{b}^{\mathrm{RGI}}\left[\left(r_{0} m_{p s}^{S}\right)^{2}-d_{b}\right] \\
& +c_{c}^{\mathrm{RGI}}\left[\left(r_{0} m_{p s}\right)^{2}-d_{c}\right]+c_{d}^{\mathrm{RGI}}\left[\left(\left(r_{0} m_{p s}^{S}\right)^{2}\right.\right. \\
& \left.\left.-2\left(r_{0} m_{p s}\right)^{2}\right) \ln \left(r_{0} m_{p s}\right)^{2}-d_{d}\right],
\end{aligned}
$$

where $d_{i}(i=b, c, d)$ can be read off from Eq. (63) and have the effect of shifting the various terms in the fit function by a constant. For example, the simplest to evaluate, $d_{b}$, is given by $\left(r_{0} m_{\pi^{+}}\right)^{2}$. Note that the fit coefficients $c_{i}^{\mathrm{RGI}}(i=b, c, d)$ are unchanged from those given in Table V. Also the numerical values of the fit function are unchanged and are given by the curves in Figs. 7-10.

Note that, although we now have to choose the scale before we make the fit, the advantage is that the error on $c_{a^{\prime}}^{\text {RGI }}$ gives directly the error on the strange quark mass up to NLO. Given this, it is no longer a disadvantage to consider the continuum extrapolation for $m_{s}^{\overline{\mathrm{MS}}}(2 \mathrm{GeV})$ directly.

As discussed earlier, we shall consider two $r_{0}$ scales. Given $\left[\Delta Z_{m}^{\overline{\mathrm{MS}}}(2 \mathrm{GeV})\right]^{-1}$ from Table I and using the experimental values of the $\pi$ and $K$ masses, Eq. (32) to determine the "pure" QCD pseudoscalar masses in Eq. (31), we find the results in Table VI.

A continuum extrapolation (using $r_{0}=0.5 \mathrm{fm}$ ) is shown in Fig. 12 together with a comparison with our previous VWI results [11]. The two methods have different $O\left(a^{2}\right)$ discretization errors, but should agree in the continuum. For the VWI method an extrapolation gave results

\footnotetext{
${ }^{10}$ It is possible to obtain expressions and hence in principle results for $f_{0},\langle\bar{q} q\rangle, 2 \alpha_{6}-\alpha_{4}$ and $2 \alpha_{8}-\alpha_{5}$ in terms of $c_{a}^{\text {RGI }}$ and $c_{i}^{\mathrm{RGI}}(i=b, c, d)$. However, these then all depend on the less well determined $\operatorname{NLO} c_{i}^{\mathrm{RGI}}(i=b, c, d)$.
} 
TABLE VI. Values of $c_{a^{\prime}}^{\mathrm{RGI}}$ and $m_{s}^{\overline{\mathrm{MS}}}(2 \mathrm{GeV})$ together with their extrapolated (continuum) values $(\beta=\infty) . \quad c_{i}^{\text {RGI }} \quad(i=$ $b, c, d)$ are given in Table V.

\begin{tabular}{|c|c|c|c|c|}
\hline \multirow[t]{2}{*}{$\beta$} & \multicolumn{2}{|c|}{$c_{a^{\prime}}^{\mathrm{RGI}}$} & \multicolumn{2}{|c|}{$m_{s}^{\overline{\mathrm{MS}}}(2 \mathrm{GeV})$} \\
\hline & $r_{0}=0.5 \mathrm{fm}$ & $r_{0}=0.467 \mathrm{fm}$ & $r_{0}=0.5 \mathrm{fm}$ & $r_{0}=0.467 \mathrm{fm}$ \\
\hline 5.20 & $0.1179(21)$ & $0.1175(21)$ & $98.71(2.33)$ & $93.35(2.29)$ \\
\hline 5.25 & $0.1233(20)$ & $0.1231(20)$ & $103.27(2.33)$ & $97.77(2.30)$ \\
\hline 5.29 & $0.1216(20)$ & $0.1214(21)$ & $101.79(2.33)$ & $96.41(2.31)$ \\
\hline 5.40 & $0.1282(18)$ & $0.1281(18)$ & $107.32(2.26)$ & $101.73(2.25)$ \\
\hline$\infty$ & $0.1393(46)$ & $0.1395(46)$ & $116.5(5.6)$ & $110.7(5.5)$ \\
\hline
\end{tabular}

of 126(5)(8) MeV, 119(5)(8) $\mathrm{MeV}$ for $r_{0}=0.5 \mathrm{fm}$ and $0.467 \mathrm{fm}$, respectively. Consistent agreement between the AWI and VWI methods within error bars is found. Finally, we quote our result for the strange quark mass

$$
m_{s}^{\overline{\mathrm{MS}}}(2 \mathrm{GeV})= \begin{cases}117(6)(4)(6) \mathrm{MeV} & \text { for } r_{0}=0.5 \mathrm{fm} \\ 111(6)(4)(6) \mathrm{MeV} & \text { for } r_{0}=0.467 \mathrm{fm}\end{cases}
$$

where the first error is statistical and the second is systematic $\approx 4 \mathrm{MeV}$. As discussed in Sec. VI A, we have determined it from the effect on $c_{a^{\prime}}^{\text {RGI }}$ of changing the fit interval. Furthermore, the additional third (systematic) error due to the $r_{0}$ scale uncertainty is $\approx 6 \mathrm{MeV}$, see Sec. II.

As discussed earlier, from the values of the $c_{a}^{\mathrm{RGI}}, c_{i}^{\mathrm{RGI}}$ $(i=b, c, d)$ coefficients we know that the corrections due to the NLO terms are small. Using the continuum value of

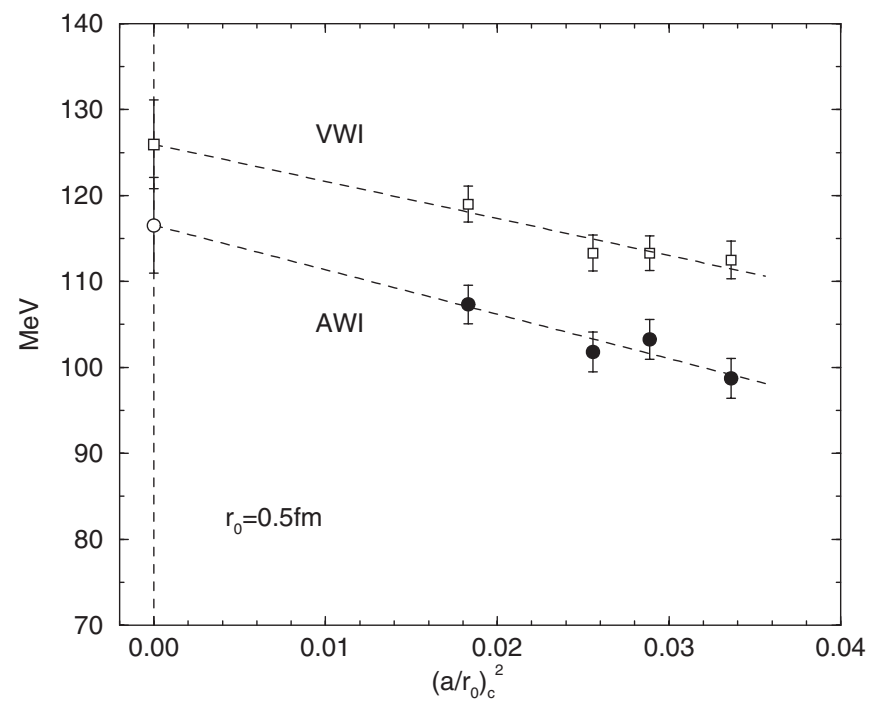

FIG. 12. $m_{s}^{\overline{\mathrm{MS}}}(2 \mathrm{GeV})$ versus $\left(a / r_{0}\right)_{c}^{2}$ (filled circles), together with a linear continuum extrapolation (empty circle). The scale used is $r_{0}=0.5 \mathrm{fm}$. For comparison we also show the results from using the VWI, open squares [11].
$c_{a}^{\mathrm{RGI}}$ from Table $\mathrm{V}$ gives for the LO strange quark mass $m_{s}^{\overline{\mathrm{MS}}}(2 \mathrm{GeV})=111(6) \mathrm{MeV}$ and $106(6) \mathrm{MeV}$ for $r_{0}=$ $0.5 \mathrm{fm}, r_{0}=0.467 \mathrm{fm}$, respectively. The difference is about $6 \mathrm{MeV}$, which means that NLO terms give about a $5 \%$ correction. We have not tried here to estimate the effects of higher order terms in $\chi \mathrm{PT},[24]$.

For the light quark mass, the numerical situation is more fortunate. From Table V, we see that $\mid\left(\left(r_{0} m_{\pi^{+}}\right)^{2}\left(c_{b}^{\mathrm{RGI}}+\right.\right.$ $\left.\left.c_{c}^{\mathrm{RGI}}\right)\right) / c_{a}^{\mathrm{RGI}} \mid \approx 0.004$ and similarly for $\mid\left(\left(r_{0} m_{\pi^{+}}\right)^{2} \times\right.$ $\left.\ln \left(r_{0} m_{\pi^{+}}\right)^{2} c_{d}^{\mathrm{RGI}} / c_{a}^{\mathrm{RGI}}\right) \mid \approx 0.005$, so corrections from LO to NLO $\chi \mathrm{PT}$ are at the $\frac{1}{2} \%$ level and are negligible here. We shall just quote the LO result of

$$
m_{u d}^{\overline{\mathrm{MS}}}(2 \mathrm{GeV})=\left\{\begin{array}{l}
4.30(25)(19)(23) \mathrm{MeV} \text { for } r_{0}=0.5 \mathrm{fm} \\
4.08(23)(19)(23) \mathrm{MeV} \text { for } r_{0}=0.467 \mathrm{fm}
\end{array}\right.
$$

where again the second error is systematic. The third (systematic) error is due to the scale $\approx 0.23 \mathrm{MeV}$.

Finally, because the NLO corrections of $\chi \mathrm{PT}$ are small, we see that the ratio

$$
\frac{m_{s}^{\overline{\mathrm{MS}}}(2 \mathrm{GeV})}{m_{u d}^{\overline{\mathrm{MS}}}(2 \mathrm{GeV})}=27.2(3.2),
$$

is close to the LO result, Eq. (33).

\section{COMPARISONS AND CONCLUSIONS}

In this article we have estimated the strange quark mass for two flavor QCD and found the result in Eq. (67), using $O(a)$ improved clover fermions and taking into consideration nonperturbative (NP) renormalization, the continuum extrapolation of the lattice results and the use of chiral perturbation theory. The NLO chiral perturbation theory yields a correction of about 5\% to the LO result, and the relevant low energy constants are in rough agreement with the phenomenological values.

It is also useful to compare our results with the results from other groups. In Fig. 13 we show some results for $n_{f}=2$ and $n_{f}=2+1$ flavors (keeping the aspect ratio approximately the same as in Fig. 12). A variety of actions, renormalizations, units and scales have been used (so the results have been plotted in physical units using the authors' preferred values). In particular the HPQCD-MILCUKQCD [8] and HPQCD [6] collaborations use improved staggered fermions. These fermions having a (remnant) chiral symmetry and are in the same situation as overlap/ domain wall fermions where there is no distinction between VWI and AWI quark masses; the bare quark mass in the Lagrangian simply needs to be renormalized.

As seen earlier in Sec. VC it is noticeable that the (tadpole-improved) perturbative results lie lower than the 


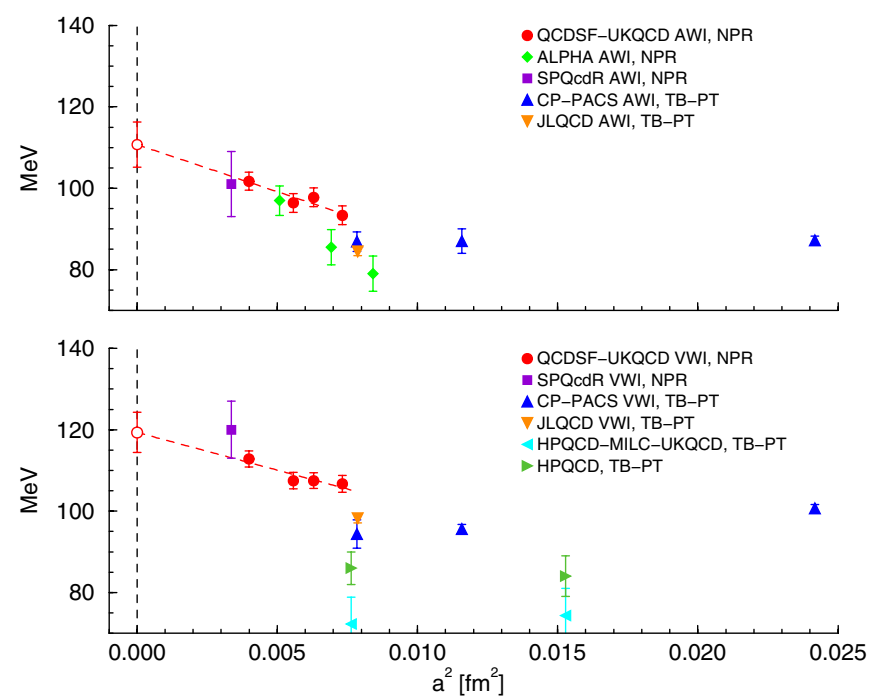

FIG. 13 (color online). Results for $m_{s}^{\overline{\mathrm{MS}}}(2 \mathrm{GeV})$ versus $a^{2}$ using the AWI (upper plot) and VWI (lower plot) methods. The results are presented with the collaborations preferred units and scales. Circles (together with a linear continuum extrapolation) are from this work and [11]; diamonds from [12]; squares from [13]; up triangles from [3]; down triangles from [4]; left triangles from [6]; right triangles from [8]. NPR denotes nonperturbative renormalization, while TB-PT denotes TI boosted perturbation theory. [6,8] are for $n_{f}=2+1$ flavors; the other results are all for $n_{f}=2$ flavors.

nonperturbatively renormalized results. Also results with $a \lesssim 0.09 \mathrm{fm}$ (i.e. $a^{2} \lesssim 0.008 \mathrm{fm}^{2}$ ) appear to be reasonably consistent with each other (this is more pronounced for the AWI results than for the VWI results). While results for $a \lesssim 0.09$ show some lattice discretization effects, using results at larger lattice spacings seems to give a fairly constant extrapolation to the continuum limit. A similar effect has also been seen elsewhere, for example, in the determination of $r_{0} \Lambda^{\overline{\mathrm{MS}}}$ for $n_{f}=0$ flavors [19], where coarse lattices also show this characteristic flattening of the data.

Finally, we compare these numbers with results from the QCD sum rule approach. A review of results from this method is given in [58], citing as a final result $m_{s}^{\overline{\mathrm{MS}}}(2 \mathrm{GeV})=99(28) \mathrm{MeV}$, while a recent five-loop calculation [59] gives $m_{s}^{\overline{\mathrm{MS}}}(2 \mathrm{GeV})=105(6)(7) \mathrm{MeV}$. These numbers cover the lattice results in Fig. 13.

In conclusion, although there is a spread of results, it would seem that the unquenched strange quark mass determined here is not lighter than the quenched strange quark mass and lies in the range of $100-130 \mathrm{MeV}$.

\section{ACKNOWLEDGMENTS}

The numerical calculations have been performed on the Hitachi SR8000 at LRZ (Munich), on the Cray T3E at EPCC (Edinburgh) [61], on the Cray T3E at NIC (Jülich) and ZIB (Berlin), as well as on the APE1000 and Quadrics at DESY (Zeuthen). We thank all institutions. This work has been supported in part by the EU Integrated Infrastructure Initiative Hadron Physics (I3HP) under Contract No. RII3-CT-2004-506078 and by the DFG under Contract No. FOR 465 (Forschergruppe Gitter-HadronenPhänomenologie).

\section{APPENDIX}

We collect here in Tables VII, VIII, IX, and X the numerical values of the partially quenched $a m_{p s}$ and the bare AWI quark terms $a \tilde{m}_{q}^{(0)}, a \tilde{m}_{q}^{(1)}$ and $a \tilde{m}_{q}$. These are defined in Eq. (39) as ratios of certain correlation functions. (The operators are summed over spatial planes, the time derivatives being taken as in footnotes ${ }^{4,6}$ ) The (bootstrap) errors for the ratios are given uniformly to two significant figures, with the overriding requirement that the result must also have a minimum of four significant figures.

TABLE VII. The bare results for $a m_{p s}, a \tilde{m}_{q}^{(0)}, a \tilde{m}_{q}^{(1)}$ and $a \tilde{m}_{q}$ for $\beta=5.20$.

\begin{tabular}{lcccc}
\hline \hline$\kappa_{q}$ & $a m_{p s}$ & $a \tilde{m}_{q}^{(0)}$ & $a \tilde{m}_{q}^{(1)}$ & $a \tilde{m}_{q}$ \\
\hline \multicolumn{5}{c}{$\kappa_{q}^{S}=0.1342$} \\
0.1334 & $0.6581(12)$ & $0.11221(19)$ & $0.22501(73)$ & $0.09778(16)$ \\
0.1338 & $0.6224(12)$ & $0.10010(19)$ & $0.20041(70)$ & $0.08724(16)$ \\
0.1342 & $0.5847(12)$ & $0.08821(19)$ & $0.17647(67)$ & $0.07689(16)$ \\
0.1347 & $0.5359(12)$ & $0.07366(19)$ & $0.14744(63)$ & $0.06420(16)$ \\
0.1353 & $0.4720(13)$ & $0.05664(19)$ & $0.11383(60)$ & $0.04934(16)$ \\
0.1356 & $0.4371(14)$ & $0.04828(19)$ & $0.09743(59)$ & $0.04203(16)$ \\
0.1360 & $0.3856(16)$ & $0.03715(22)$ & $0.07576(60)$ & $0.03229(19)$ \\
0.1362 & $0.3569(17)$ & $0.03159(25)$ & $0.06515(64)$ & $0.02741(22)$ \\
0.5 & $\kappa_{q}^{S}=0.1350$ \\
0.1332 & $0.5985(11)$ & $0.10076(16)$ & $0.18529(60)$ & $0.08888(13)$ \\
0.1337 & $0.5515(11)$ & $0.08566(16)$ & $0.15670(58)$ & $0.07561(13)$ \\
0.1342 & $0.5018(11)$ & $0.07088(15)$ & $0.12923(55)$ & $0.06260(13)$ \\
0.1345 & $0.4703(12)$ & $0.06218(15)$ & $0.11327(54)$ & $0.05492(13)$ \\
0.1350 & $0.4148(13)$ & $0.04785(15)$ & $0.08755(54)$ & $0.04224(13)$ \\
0.1353 & $0.3771(15)$ & $0.03954(17)$ & $0.07246(54)$ & $0.03490(14)$ \\
0.1355 & $0.3505(19)$ & $0.03397(18)$ & $0.06256(57)$ & $0.02996(16)$ \\
0.1357 & $0.3216(20)$ & $0.02837(20)$ & $0.05293(57)$ & $0.02497(17)$ \\
& & \multicolumn{5}{c}{$\kappa_{q}^{S}=0.1355$} \\
0.1332 & $0.5546(10)$ & $0.09121(14)$ & $0.15890(54)$ & $0.08102(12)$ \\
0.1336 & $0.5158(11)$ & $0.07911(14)$ & $0.13707(52)$ & $0.07032(13)$ \\
0.1340 & $0.4751(11)$ & $0.06718(15)$ & $0.11600(51)$ & $0.05974(13)$ \\
0.1343 & $0.4430(12)$ & $0.05842(15)$ & $0.10069(50)$ & $0.05196(13)$ \\
0.1348 & $0.3848(14)$ & $0.04410(15)$ & $0.07610(51)$ & $0.03922(14)$ \\
0.1350 & $0.3600(15)$ & $0.03847(15)$ & $0.06656(51)$ & $0.03421(14)$ \\
0.1353 & $0.3200(17)$ & $0.03014(16)$ & $0.05252(53)$ & $0.02677(14)$ \\
0.1355 & $0.2907(15)$ & $0.02451(15)$ & $0.04275(43)$ & $0.02177(13)$ \\
0.1357 & $0.2577(23)$ & $0.01909(18)$ & $0.03475(51)$ & $0.01687(16)$ \\
\hline \hline
\end{tabular}


ESTIMATING THE UNQUENCHED STRANGE QUARK ...

TABLE VIII. The bare results for $a m_{p s}, a \tilde{m}_{q}^{(0)}, a \tilde{m}_{q}^{(1)}$ and $a \tilde{m}_{q}$ for $\beta=5.25$.

\begin{tabular}{lcccc}
\hline \hline$\kappa_{q}$ & $a m_{p s}$ & $a \tilde{m}_{q}^{(0)}$ & $a \tilde{m}_{q}^{(1)}$ & $a \tilde{m}_{q}$ \\
\hline \multicolumn{5}{c}{$\kappa_{q}^{S}=0.1346$} \\
0.1337 & $0.5794(15)$ & $0.09697(19)$ & $0.17225(87)$ & $0.08723(17)$ \\
0.1340 & $0.5514(15)$ & $0.08793(19)$ & $0.15554(86)$ & $0.07914(17)$ \\
0.1346 & $0.4932(10)$ & $0.07027(12)$ & $0.12399(47)$ & $0.06326(11)$ \\
0.1349 & $0.4612(17)$ & $0.06148(18)$ & $0.10782(79)$ & $0.05539(17)$ \\
0.1353 & $0.4168(18)$ & $0.05005(18)$ & $0.08774(76)$ & $0.04509(17)$ \\
0.1355 & $0.3932(18)$ & $0.04440(18)$ & $0.07794(75)$ & $0.03999(17)$ \\
0.1359 & $0.3420(20)$ & $0.03317(19)$ & $0.05877(64)$ & $0.02985(18)$ \\
0.1361 & $0.3133(22)$ & $0.02750(21)$ & $0.04913(65)$ & $0.02472(19)$ \\
\multicolumn{5}{c}{$\kappa_{q}^{S}=0.1352$} \\
0.1337 & $0.5419(11)$ & $0.08860(16)$ & $0.15186(60)$ & $0.08002(14)$ \\
0.1341 & $0.5027(12)$ & $0.07658(16)$ & $0.13073(57)$ & $0.06919(14)$ \\
0.1345 & $0.4621(13)$ & $0.06474(16)$ & $0.11027(54)$ & $0.05851(14)$ \\
0.1348 & $0.4300(13)$ & $0.05599(16)$ & $0.09536(52)$ & $0.05060(14)$ \\
0.1352 & $0.3821(13)$ & $0.04432(12)$ & $0.07471(38)$ & $0.04010(10)$ \\
0.1355 & $0.3466(17)$ & $0.03593(17)$ & $0.06178(50)$ & $0.03244(16)$ \\
0.1358 & $0.3054(20)$ & $0.02740(19)$ & $0.04775(53)$ & $0.02470(18)$ \\
0.1359 & $0.2901(22)$ & $0.02452(21)$ & $0.04309(56)$ & $0.02209(20)$ \\
0.1336 & $0.50970(72)$ & $0.084021(81)$ & $0.13257(36)$ & $0.076528(72)$ \\
0.1339 & $0.48011(72)$ & $0.074990(81)$ & $0.11733(35)$ & $0.068359(72)$ \\
0.1343 & $0.43883(73)$ & $0.063110(80)$ & $0.09772(33)$ & $0.057587(73)$ \\
0.1346 & $0.40619(74)$ & $0.054323(81)$ & $0.08354(31)$ & $0.049601(74)$ \\
0.1350 & $0.35966(76)$ & $0.042776(83)$ & $0.06532(28)$ & $0.039085(76)$ \\
0.1352 & $0.33469(77)$ & $0.037071(84)$ & $0.05657(24)$ & $0.033874(78)$ \\
0.1355 & $0.29421(81)$ & $0.028608(87)$ & $0.04360(22)$ & $0.026144(81)$ \\
0.13575 & $0.25556(55)$ & $0.021495(57)$ & $0.03291(15)$ & $0.019635(52)$ \\
0.1360 & $0.2117(13)$ & $0.01456(11)$ & $0.02256(26)$ & $0.013281(98)$ \\
\hline \hline
\end{tabular}

The second column in the tables gives the pion mass, defined in the standard way from the correlation function

$$
\left\langle P^{\text {smeared }}(t) P^{\text {smeared }}(0)\right\rangle \stackrel{t \gg 0}{=} A\left(e^{-m_{p s} t}+e^{-m_{p s}\left(a N_{T}-t\right)}\right),
$$

where the correlation function is evaluated in a configuration with sea quark mass $a m_{q}^{S}$. (The normalization is not important here so we can work with unimproved operators to obtain the pseudoscalar masses.) The smearing used is Jacobi smearing (see e.g. [60]), with typical parameters $\kappa_{s}=0.21$ and $n_{s}=50$.

The third and fourth columns in the tables give the bare results for $a \tilde{m}_{q}^{(0)}$ and $a \tilde{m}_{q}^{(1)}$, as defined in Eq. (39).

The improvement coefficient $c_{A}$ has been determined nonperturbatively in [20]. We use the values obtained there of

$$
\begin{array}{cc}
\beta & c_{A} \\
5.20 & -0.0641(40) \\
5.25 & -0.0565(40) \\
5.29 & -0.0517(40) \\
5.40 & -0.0420(40)
\end{array}
$$

\begin{tabular}{|c|c|c|c|c|}
\hline$\kappa_{q}$ & $a m_{p s}$ & $a \tilde{m}_{q}^{(0)}$ & $a \tilde{m}_{q}^{(1)}$ & $a \tilde{m}_{q}$ \\
\hline \multicolumn{5}{|c|}{$\kappa_{q}^{S}=0.1340$} \\
\hline 0.1340 & $0.5767(11)$ & $0.09689(19)$ & 0.171 & $0.08802(17)$ \\
\hline 1344 & $0.5392(15)$ & $0.08480(18)$ & $1(82)$ & 0.077 \\
\hline .1349 & 0.4901 & 0.0701 & $0.12204(81)$ & 0.063 \\
\hline 52 & 0.4589 & 0.061 & 0.106 & 0.05 \\
\hline 1355 & $0.4255(17)$ & 0.0528 & $0.09169(80)$ & $0.04809(17)$ \\
\hline 0.1357 & $0.4024(20)$ & 0.0471 & $0.08189(80)$ & $0.04292(17)$ \\
\hline .1359 & 0.378 & 0.0415 & 0.072 & 0.037 \\
\hline 1362 & 0.3384 & $0.03315(23)$ & $0.05830(81)$ & $0.03013(21)$ \\
\hline \multicolumn{5}{|c|}{$\kappa_{q}^{S}=0.1350$} \\
\hline 340 & 0.5222 & $0.08542(11)$ & 0.140 & 0.078 \\
\hline 343 & $0.4 \mathrm{C}$ & 0.076 & 0.12 & 0.06 \\
\hline 347 & 0.4 & 0.06 & & 0.05 \\
\hline 0.1350 & $0.4205^{\circ}$ & 0.055 & 0.0 & 0.051 \\
\hline 0.1355 & & 0.041 & & 0.03 \\
\hline & 0.338 & 0.035 & $0.05^{7}$ & 0.032 \\
\hline 0.1360 & 0.296 & 0.027 & 0.04 & 0.02 \\
\hline 0.1361 & 0.27 & $0.02427(15)$ & 0.039 & 0.022 \\
\hline \multicolumn{5}{|c|}{$\kappa_{q}^{S}=0.1355$} \\
\hline 39 & 0.49 & $0.08260(11)$ & $0(44)$ & 0.07 \\
\hline .1343 & 0.461 & 0.070 & 0.108 & 0.06 \\
\hline 0.1346 & 0.430 & 0.061 & 0.094 & 0.05 \\
\hline 49 & 0.3977 & 0.052 & 0.08 & 0.048 \\
\hline 0.1353 & $0.35144(91)$ & 0.041 & 0.062 & 0.038 \\
\hline 0.1355 & 0.326 & 0.035 & 0.054 & 0.032 \\
\hline 0.1358 & $0.2858(11)$ & $0.02723(11)$ & 0.041 & 0.025 \\
\hline 0.1360 & 0.255 & 0.0215 & $0.03316(31)$ & 0.019 \\
\hline 0.1363 & $0.2012(18)$ & $0.01304(14)$ & $0.02056(30)$ & $0.01197(13)$ \\
\hline \multicolumn{5}{|c|}{$\boldsymbol{\kappa}_{q}^{S}=0.1359$} \\
\hline 0.1339 & $0.47757(65)$ & $0.078462(92)$ & $0.11620(29)$ & $0.072456(88)$ \\
\hline 0.13425 & $0.44247(66)$ & $0.067952(92)$ & $0.09946(28)$ & $0.062811(88)$ \\
\hline 0.1346 & $0.40540(70)$ & 0.0575 & $3(27)$ & $0.053259(88)$ \\
\hline 0.13505 & $0.35469(70)$ & $0.044401(93)$ & $0.06350(25)$ & $0.041119(89)$ \\
\hline & $0.32287(73)$ & $0.036894(94)$ & $0.05251(24)$ & $0.034180(90)$ \\
\hline 0.13562 & $0.28151(78)$ & $0.028036(96)$ & $0.03982(23)$ & $0.025977(91)$ \\
\hline 0.1359 & $0.23924(87)$ & $0.020134(92)$ & $0.02886(21)$ & $0.018642(86)$ \\
\hline 0.13617 & $0.1899(12)$ & $0.01239(10)$ & $0.01837(25)$ & $0.011444(98)$ \\
\hline
\end{tabular}

PHYSICAL REVIEW D 73, 054508 (2006)

TABLE IX. The bare results for $a m_{p s}, a \tilde{m}_{q}^{(0)}, a \tilde{m}_{q}^{(1)}$ and $a \tilde{m}_{q}$ for $\beta=5.29$.

From the tables it can be seen that the inclusion of the improvement term $\left(\times c_{A}\right)$ to the quark mass gives a noticeable change in the final result. Also the error in $c_{A}$ has an effect. Although not a large difference to using simple error propagation for the three quantities $\left(a \tilde{m}_{q}^{(0)}, a \tilde{m}_{q}^{(1)}\right.$ and $c_{A}$ ), to try to minimize the error propagation we have adopted the procedure of first finding the bootstrap error for $a \tilde{m}_{q}^{(0)}+c_{A} a \tilde{m}_{q}^{(1)}$ (with fixed $c_{A}$ ) and then including the independent error of $c_{A}(\times$ fixed $a \tilde{m}_{q}^{(1)}$ ) by error propagation. This gives the results for $a \tilde{m}_{q}$ shown in the fifth column of the tables. 
TABLE X. The bare results for $a m_{p s}, a \tilde{m}_{q}^{(0)}, a \tilde{m}_{q}^{(1)}$ and $a \tilde{m}_{q}$ for $\beta=5.40$.

\begin{tabular}{|c|c|c|c|c|}
\hline$\kappa_{q}$ & $a m_{p s}$ & $a \tilde{m}_{q}^{(0)}$ & $a \tilde{m}_{q}^{(1)}$ & $a \tilde{m}_{q}$ \\
\hline \multicolumn{5}{|c|}{$\kappa_{q}^{S}=0.1350$} \\
\hline 0.1346 & $0.44399(52)$ & $0.071849(57)$ & $0.10023(25)$ & $0.067635(52)$ \\
\hline 0.1350 & $0.40301(43)$ & $0.059913(50)$ & $0.08250(18)$ & $0.056444(47)$ \\
\hline 0.1353 & $0.37156(54)$ & $0.051132(70)$ & $0.06996(21)$ & $0.048190(67)$ \\
\hline 0.1357 & $0.32541(63)$ & $0.039519(68)$ & $0.05359(21)$ & $0.037266(64)$ \\
\hline 0.13602 & $0.28482(69)$ & $0.030274(70)$ & $0.04100(21)$ & $0.028550(66)$ \\
\hline 0.1363 & $0.24504(77)$ & $0.022220(72)$ & $0.03027(20)$ & $0.020947(68)$ \\
\hline 0.13655 & $0.20349(95)$ & $0.014975(74)$ & $0.02098(22)$ & $0.014093(70)$ \\
\hline \multicolumn{5}{|c|}{$\boldsymbol{\kappa}_{q}^{S}=0.1356$} \\
\hline 0.1346 & $0.42009(66)$ & $0.067720(51)$ & $0.08973(29)$ & $0.063947(47)$ \\
\hline 0.13494 & $0.38581(63)$ & $0.057620(49)$ & $0.07533(28)$ & $0.054453(45)$ \\
\hline 0.1353 & $0.34617(72)$ & $0.046987(49)$ & $0.06068(26)$ & $0.044436(46)$ \\
\hline 0.1356 & $0.31232(67)$ & $0.038239(49)$ & $0.04926(22)$ & $0.036168(44)$ \\
\hline 0.13591 & $0.27210(77)$ & $0.029197(52)$ & $0.03725(24)$ & $0.027631(50)$ \\
\hline 0.13618 & $0.23346(87)$ & $0.021380(56)$ & $0.02742(23)$ & $0.020227(53)$ \\
\hline 0.13643 & $0.1921(10)$ & $0.014081(68)$ & $0.01873(22)$ & $0.013293(65)$ \\
\hline \multicolumn{4}{|c|}{$\kappa_{q}^{S}=0.1361$} & $0.01116(13)$ \\
\hline 0.1346 & $0.40055(60)$ & $0.064373(49)$ & $0.08174(23)$ & $0.060936(45)$ \\
\hline 0.13493 & $0.36621(63)$ & $0.054572(47)$ & $0.06820(22)$ & $0.051704(44)$ \\
\hline 0.13525 & $0.33068(70)$ & $0.045072(53)$ & $0.05559(21)$ & $0.042735(50)$ \\
\hline 0.13555 & $0.29521(76)$ & $0.036298(51)$ & $0.04425(20)$ & $0.034437(49)$ \\
\hline 0.13584 & $0.25784(85)$ & $0.027842(55)$ & $0.03372(20)$ & $0.026425(53)$ \\
\hline 0.1361 & $0.22081(72)$ & $0.020335(47)$ & $0.02455(16)$ & $0.019303(44)$ \\
\hline 0.13632 & $0.1833(12)$ & $0.013937(71)$ & $0.01705(22)$ & $0.013220(68)$ \\
\hline 0.1364 & $0.1668(19)$ & $0.011552(88)$ & $0.01457(22)$ & $0.010940(84)$ \\
\hline
\end{tabular}

[1] T. Bakeyev, M. Göckeler, R. Horsley, D. Pleiter, P. E. L. Rakow, G. Schierholz, and H. Stüben (QCDSF-UKQCD Collaboration), Phys. Lett. B 580, 197 (2004).

[2] A. Ali Khan, S. Aoki, G. Boyd, R. Burkhalter, S. Ejiri, M. Fukugita, S. Hashimoto, N. Ishizuka, Y. Iwasaki, K. Kanaya, T. Kaneko, Y. Kuramashi, T. Manke, K. Nagai, M. Okawa, H. P. Shanahan, A. Ukawa, and T. Yoshié (CPPACS Collaboration), Phys. Rev. Lett. 85, 4674 (2000); 90, 029902(E) (2003).

[3] A. Ali Khan, S. Aoki, G. Boyd, R. Burkhalter, S. Ejiri, M. Fukugita, S. Hashimoto, N. Ishizuka, Y. Iwasaki, K. Kanaya, T. Kaneko, Y. Kuramashi, T. Manke, K. Nagai, M. Okawa, H. P. Shanahan, A. Ukawa, and T. Yoshié (CPPACS Collaboration), Phys. Rev. D 65, 054505 (2002); 67, 059901(E) (2003).

[4] S. Aoki, R. Burkhalter, M. Fukugita, S. Hashimoto, K-I. Ishikawa, N. Ishizuka, Y. Iwasaki, K. Kanaya, T. Kaneko, Y. Kuramashi, M. Okawa, T. Onogi, N. Tsutsui, A. Ukawa, N. Yamada, and T. Yoshié (JLQCD Collaboration), Phys. Rev. D 68, 054502 (2003).

[5] T. Kaneko, S. Aoki, M. Fukugita, S. Hashimoto, K-I. Ishikawa, T. Ishikawa, N. Ishizuka, Y. Iwasaki, K. Kanaya, Y. Kuramashi, M. Okawa, N. Taniguchi, N. Tsutsui, A. Ukawa, and T. Yoshié (CP-PACS Collaboration), Nucl. Phys. B, Proc. Suppl. 129, 188 (2004).
[6] C. Aubin, C. Bernard, C. Davies, C. DeTar, S. Gottlieb, A. Gray, E. Gregory, J. Hein, U. Heller, J. Hetrick, G. P. Lepage, Q. Mason, J. Osborn, J. Shigemitsu, R. Sugar, D. Toussaint, H. Trottier, and M. Wingate (HPQCDMILC-UKQCD Collaboration), Phys. Rev. D 70, 031504 (2004).

[7] T. Ishikawa, S. Aoki, O. Bär, M. Fukugita, S. Hashimoto, K.-I. Ishikawa, N. Ishizuka, Y. Iwasaki, K. Kanaya, T. Kaneko, Y. Kuramashi, M. Okawa, Y. Taniguchi, N. Tsutsui, A. Ukawa, and T. Yoshié (CP-PACS-JLQCD Collaboration), Proc. of Sci., LAT2005 (2005) 057 [heplat/0509142].

[8] Q. Mason, H. D. Trottier, R. Horgan, C. T. H. Davies, and G. P. Lepage (HPQCD Collaboration), hep-ph/0511160.

[9] M. Göckeler, R. Horsley, H. Oelrich, D. Petters, D. Pleiter, P. E. L. Rakow, G. Schierholz, and P. Stephenson (QCDSF Collaboration), Phys. Rev. D 62, 054504 (2000).

[10] J. Garden, J. Heitger, R. Sommer, and H. Wittig (ALPHAUKQCD Collaboration), Nucl. Phys. B571, 237 (2000).

[11] M. Göckeler, R. Horsley, A. C. Irving, D. Pleiter, P. E. L. Rakow, G. Schierholz, and H. Stüben (QCDSF-UKQCD Collaboration), hep-ph/0409312.

[12] M. Della Morte, R. Hoffmann, F. Knechtli, J. Rolf, R. Sommer, I. Wetzorke, and U. Wolff (ALPHA Collaboration), Nucl. Phys. B729, 117 (2005).

[13] D. Bećirević, B. Blossier, P. Boucaud, V. Giménez, V. 
Lubicz, F. Mescia, S. Simula, and C. Tarantino (SPQcdR Collaboration), Nucl. Phys. B734, 138 (2006).

[14] O. V. Tarasov, A. A. Vladimirov, and A. Yu. Zharkov, Phys. Lett. 93B, 429 (1980).

[15] S. A. Larin and J. A. M. Vermaseren, Phys. Lett. B 303, 334 (1993).

[16] T. van Ritbergen, J.A. M. Vermaseren, and S. A. Larin, Phys. Lett. B 400, 379 (1997).

[17] K. G. Chetyrkin, Phys. Lett. B 404, 161 (1997).

[18] J. A. M. Vermaseren, S. A. Larin, and T. van Ritbergen, Phys. Lett. B 405, 327 (1997).

[19] M. Göckeler, R. Horsley, A. C. Irving, D. Pleiter, P. E. L. Rakow, G. Schierholz, and H. Stüben (QCDSF-UKQCD Collaboration), Phys. Rev. D 73, 014513 (2006).

[20] M. Della Morte, R. Frezzotti, J. Heitger, J. Rolf, R. Sommer, and U. Wolff (ALPHA Collaboration), Nucl. Phys. B713, 378 (2005).

[21] A. Ali Khan, T. Bakeyev, M. Göckeler, T. R. Hemmert, R. Horsley, A. C. Irving, B. Joó, D. Pleiter, P. E. L. Rakow, G. Schierholz, and H. Stüben (QCDSF-UKQCD Collaboration), Nucl. Phys. B689, 175 (2004).

[22] C. Aubin, C. Bernard, C. DeTar, S. Gottlieb, E. B. Gregory, U. M. Heller, J. E. Hetrick, J. Osborn, R. Sugar, and D. Toussaint, Phys. Rev. D 70, 094505 (2004).

[23] M. Göckeler, R. Horsley, D. Pleiter, P.E. L. Rakow, G. Schierholz, W. Schroers, H. Stüben, and J. M. Zanotti (QCDSF-UKQCD Collaboration), Proc. of Sci., LAT2005 (2005) 063 [hep-lat/0509196].

[24] J. Bijnens and T. A. Lähde, Phys. Rev. D 72, 074502 (2005).

[25] C. Bernard and M. Golterman, Phys. Rev. D 49, 486 (1994).

[26] S. R. Sharpe, Phys. Rev. D 56, 7052 (1997); 62, 099901(E) (2000).

[27] J. Heitger, R. Sommer, and H. Wittig, Nucl. Phys. B588, 377 (2000).

[28] M. Jamin, Phys. Lett. B 538, 71 (2002).

[29] T-P. Cheng and L-F. Li, Gauge Theory of Elementary Particle Physics (Oxford University Press, New York, 1984).

[30] H. Leutwyler, Nucl. Phys. B, Proc. Suppl. 94, 108 (2001).

[31] J. Bijnens, Phys. Lett. B 306, 343 (1993).

[32] R. Baur and R. Urech, Phys. Rev. D 53, 6552 (1996).

[33] H. Leutwyler, Phys. Lett. B 378, 313 (1996).

[34] B. Ananthanarayan and B. Moussallam, J. High Energy Phys. 06 (2004) 047.

[35] J. Bijnens and J. Prades, Nucl. Phys. B490, 239 (1997).

[36] J.F. Donoghue and A.F. Perez, Phys. Rev. D 55, 7075 (1997).

[37] Y. Namekawa and Y. Kikukawa, Proc. of Sci., LAT2005 (2005) 090 [hep-lat/0509120].
[38] N. Yamada, T. Blum, M. Hayakawa, and T. Izubuchi (RBC Collaboration), Proc. of Sci., LAT2005 (2005) 092 [heplat/0509124].

[39] M. Della Morte, R. Hoffmann, and R. Sommer, J. High Energy Phys. 03 (2005) 029.

[40] S. Sint and P. Weisz, Nucl. Phys. B502, 251 (1997).

[41] M. Guagnelli, R. Petronzio, J. Rolf, S. Sint, R. Sommer, and U. Wolff (ALPHA Collaboration), Nucl. Phys. B595, 44 (2001).

[42] M. Göckeler, R. Horsley, D. Pleiter, P.E. L. Rakow, A. Schäfer, G. Schierholz, H. Stüben, and J.M. Zanotti (QCDSF Collaboration), Phys. Rev. D 72, 054507 (2005).

[43] M. Göckeler, R. Horsley, D. Pleiter, P. E. L. Rakow, and G. Schierholz (QCDSF Collaboration), Phys. Rev. D 71, 114511 (2005).

[44] G. Martinelli, C. Pittori, C. T. Sachrajda, M. Testa, and A. Vladikas, Nucl. Phys. B445, 81 (1995).

[45] M. Göckeler, R. Horsley, H. Oelrich, H. Perlt, D. Petters, P. E. L. Rakow, A. Schäfer, G. Schierholz, and A. Schiller (QCDSF Collaboration), Nucl. Phys. B544, 699 (1999).

[46] E. Franco and V. Lubicz, Nucl. Phys. B531, 641 (1998).

[47] K. G. Chetyrkin and A. Rétey, Nucl. Phys. B583, 3 (2000).

[48] K. G. Chetyrkin and A. Rétey, hep-ph/0007088.

[49] J. R. Cudell, A. Le Yaouanc, and C. Pittori, Phys. Lett. B 454, 105 (1999).

[50] K. D. Lane, Phys. Rev. D 10, 2605 (1974).

[51] H. Pagels, Phys. Rev. D 19, 3080 (1979).

[52] D. Bécirević and V. Lubicz, Phys. Lett. B 600, 83 (2004).

[53] L. Giusti and A. Vladikas, Phys. Lett. B 488, 303 (2000).

[54] S. Capitani, M. Göckeler, R. Horsley, D. Pleiter, P. Rakow, H. Stüben, and G. Schierholz (QCDSF Collaboration), Nucl. Phys. B, Proc. Suppl. 106, 299 (2002).

[55] S. Capitani, M. Göckeler, R. Horsley, H. Perlt, P E. L. Rakow, G. Schierholz, and A. Schiller (QCDSF Collaboration), Nucl. Phys. B593, 183 (2001).

[56] M. Della Morte, R. Hoffmann, F. Knechtli, R. Sommer, and U. Wolff (ALPHA Collaboration), J. High Energy Phys. 07 (2005) 007.

[57] C. McNeile, Phys. Lett. B 619, 124 (2005).

[58] S. Narison, hep-ph/0510108.

[59] K. G. Chetyrkin and A. Khodjamirian, hep-ph/0512295.

[60] C. Best, M. Göckeler, R. Horsley, E.-M. Ilgenfritz, H. Perlt, P. Rakow, A. Schäfer, G. Schierholz, A. Schiller, and S. Schramm (QCDSF Collaboration), Phys. Rev. D 56, 2743 (1997).

[61] C. R. Allton, S. P. Booth, K. C. Bowler, J. Garden, A. Hart, D. Hepburn, A. C. Irving, B. Joó, R. D. Kenway, C. M. Maynard, C. McNeile, C. Michael, S. M. Pickles, J. C. Sexton, K. J. Sharkey, Z. Sroczynski, M. Talevi, M. Teper, and H. Wittig (UKQCD Collaboration), Phys. Rev. D 65, 054502 (2002). 